\title{
A Limit Theorem for Birkhoff Sums of non-Integrable Functions over Rotations
}

\author{
Yakov G. Sinai and Corinna Ulcigrai \\ Dedicated to M. Brin on the occasion of his sixtieth birthday.
}

\begin{abstract}
We consider Birkhoff sums of functions with a singularity of type $1 / x$ over rotations and prove the following limit theorem. Let $S_{N}=S_{N}(\alpha, x)$ be the $N^{t h}$ non-renormalized Birkhoff sum, where $\alpha \in[0,1)$ is the rotation number, $x \in[0,1)$ is the initial point and $(\alpha, x)$ are uniformly distributed. We prove that $S_{N} / N$ has a joint limiting distribution in $(\alpha, x)$ as $N$ tends to infinity. As a corollary, we get the existence of a limiting distribution for certain trigonometric sums.
\end{abstract}

The purpose of this paper is the proof of the following theorem.

Theorem 1. For any Borel-measurable subset $\Omega \subset \mathbb{C}$ there exists

$$
\lim _{N \rightarrow \infty} \operatorname{Leb}\left\{(\alpha, x): \frac{1}{N} \sum_{n=0}^{N-1} \frac{1}{1-e^{2 \pi i(n \alpha+x)}} \in \Omega .\right\}=\mathbb{P}(\Omega)
$$

Here Leb denotes the two-dimensional Lebesgue measure on $[0,1) \times[0,1)$ and $\mathbb{P}$ is a probability measure on $\mathbb{C}$.

In other words, the trigonometric sums $\frac{1}{N} \sum_{n=0}^{N-1}\left(1-e^{2 \pi i(n \alpha+x)}\right)^{-1}$ have a limiting distribution.

The theorem follows as a corollary from the following more general theorem.

Let $R_{\alpha}(x)=x+\alpha(\bmod 1)$ be the rotation by $\alpha \in \mathbb{R}$ on $[0,1)$. Let $f(x)=$ $f_{1}(x)+f_{2}(x)$ where

(i) $f_{1}: \mathbb{R} \backslash \mathbb{Z} \rightarrow \mathbb{R}$ is periodic of period 1 and $C^{1}$ on $\mathbb{R} \backslash \mathbb{Z}$;

(ii) $f_{1}(x)=\frac{c}{x}$ on $[\epsilon, 0) \cup(0, \epsilon]$ for some $\epsilon<1$ and $c \neq 0$;

(iii) $f_{2}$ is a 1 -periodic function, which extends to a $C^{1}$ function on $[0,1]$.

2000 Mathematics Subject Classification. Primary 37A30: Secondary 37E10, 60B10.

Key words and phrases. Limit theorems, Rotations, Birkhoff Sums, Principal Value, Continued Fraction.

The first author thanks NSF Grant DMS 0600996 for the financial support.

The second author thanks the Clay Mathematics Institute, since part of this work was completed while she was supported by a Liftoff Fellowship.

(C)0000 (copyright holder) 
Theorem 2. For any $a<b$ there exists the limit

$$
\lim _{N \rightarrow \infty} \operatorname{Leb}\left\{(\alpha, x): a \leq \frac{1}{N} \sum_{n=0}^{N-1} f\left(R_{\alpha}^{n} x\right) \leq b\right\}=P(a, b),
$$

where $P$ is a probability measure on $\mathbb{R}$.

In other words, the random variables $X_{N}:=\frac{1}{N} \sum_{n=0}^{N-1} f\left(R_{\alpha}^{n} x\right)$ considered as functions of $\alpha$ and $x$ have a limiting distribution.

Using periodicity of $f_{1}$ and redefining $f_{2}$ appropriately, we can replace (ii) by $(i i)^{\prime}$ :

$$
\text { (ii)' } f_{1}(x)=\frac{c}{x}-\frac{c}{1-x} \text { for } 0<x<1 .
$$

In what follows we will assume that $f_{1}$ and $f_{2}$ satisfy $(i),(i i)^{\prime}$ and (iii).

Theorem 1 follows from Theorem 2. Indeed, splitting into real and imaginary part, we can write

$$
\frac{1}{1-e^{2 \pi i(n \alpha+x)}}=\frac{1}{2}+\frac{i}{2} f\left(R_{\alpha}^{n} x\right), \quad f(x):=\frac{\sin 2 \pi x}{1-\cos 2 \pi x} .
$$

Then $f(x)$ satisfies $(i),(i i)^{\prime}$ (with $c=\frac{1}{2 \pi}$ ) and (iii). Hence Theorem 1 is a corollary of Theorem 2

Let us use the notation

$$
S_{N}(\alpha, x, f)=S_{N}(\alpha, x):=\sum_{n=0}^{N-1} f\left(R_{\alpha}^{n} x\right)
$$

for the $N^{t h}$ non-normalized Birkhoff sum of the function $f$ under $R_{\alpha}$. The dependence on $f$ will be omitted if there is no ambiguity. Similar theorems can be proved for expressions of the form

$$
\frac{1}{2 N+1} \sum_{n=-N}^{N} \frac{1}{e^{2 \pi i(n \alpha+x)}-1} \text { and } \frac{1}{2 N+1} \sum_{n=-N}^{N} f\left(R_{\alpha}^{n} x\right) .
$$

Another example of Birkhoff sums with this type of singularity is given by the trigonometric series of cosecants, i.e. $\sum_{n=1}^{\infty} \sin (n \pi \alpha)^{-1}$. This series was investigated by Hardy and Littlewood in HL30, where they prove in particular that when $\alpha$ is a quadratic irrational, the corresponding partial sums are uniformly bounded.

Outline of the proof. The strategy of the proof is the following. For any positive $\epsilon$ and $\delta$ we construct approximate sums $G_{N}^{\epsilon, \delta}$, which are close to $S_{N}(\alpha, x)$ in probability, i.e. for all sufficiently large $N$

$$
\operatorname{Leb}\left\{(\alpha, x):\left|G_{N}^{\epsilon, \delta}-\frac{1}{N} S_{N}(\alpha, x)\right| \geq \epsilon\right\} \leq \delta .
$$

Then we prove that, for each $\epsilon$ and $\delta, G_{N}^{\epsilon, \delta}$ has a limiting distribution as $N \rightarrow \infty$ and the distributions of $G_{N}^{\epsilon, \delta}$ are weakly compact in $N, \epsilon$ and $\delta$. All these statements together allow to prove Theorem 2 ,

Our strategy is to show that $G_{N}^{\epsilon, \delta}$ can be expressed as functions of quantities which do have a limiting distribution. In particular, one of the quantities involved is the ratio $q_{n(N)} / N$ where $q_{n}$ are denominators of the continued fraction expansion of $\alpha$ and $n(N)$ is determined by $q_{n(N)} \leq N<q_{n(N)+1}$. We use the renewal-type 

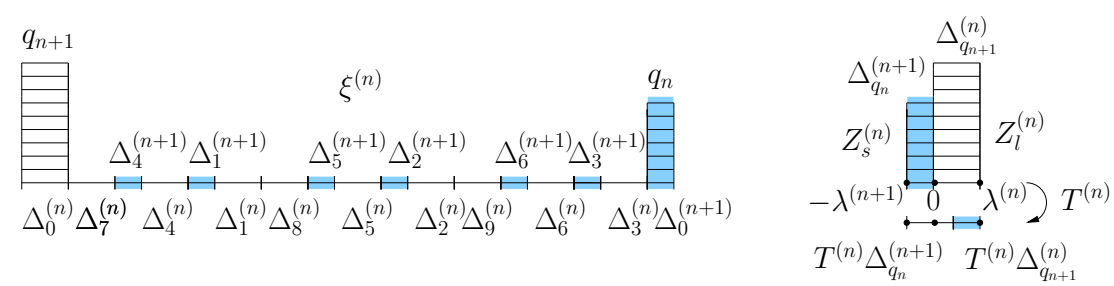

FiguRE 1. The partition $\xi^{(n)}$ and its representation into towers $Z_{l}^{(n)}$ and $Z_{s}^{(n)}$.

limit theorem proved in SU08 which gives the existence of a limiting distribution for the ratio $q_{n(N)} / N$. This theorem is recalled and generalized in $\$ 1.2$,

The other basic tool is the classical system of partitions of the unit circle induced by the continued fraction expansion (whose definition is recalled in \$1.1). Using this system of partitions, the Birkhoff sums in (0.2) are decomposed onto simpler orbit segments, which we call cycles and analyze separately in $₫ 2$. The key phenomenon which implies the asymptotic behavior of the Birkhoff sums is the cancellation between positive and negative contributions to each cycle (see 2.2 ) which resemble the existence of the principal value in non-absolutely converging integrals. The decomposition into cycles is explained in 93 . The proof of Theorem 2 is given in 4

\section{Preliminaries.}

1.1. Continued fractions and partitions of the interval. The following system of partitions exists for any $R_{\alpha}$ with irrational $\alpha$ (see, e.g. Sin94). Write down the expansion of $\alpha$ as a continued fraction:

$$
\alpha=\left[a_{1}, a_{2}, \ldots, a_{n}, \ldots\right]
$$

and let $\alpha_{n}=\frac{p_{n}}{q_{n}}=\left[a_{1}, a_{2}, \ldots, a_{n}\right]$ be the $n^{t h}$ approximant. Let $\{x\}$ be the fractional part of $x$. Denote by

$$
\Delta^{(n)}:=\Delta_{0}^{(n)}= \begin{cases}{\left[0,\left\{q_{n} \alpha\right\}\right)} & \text { if } n \text { is even; } \\ {\left[\left\{q_{n} \alpha\right\}, 1\right)} & \text { if } n \text { is odd. }\end{cases}
$$

For $n$ even, the intervals $\Delta^{(n)}$ and $\Delta^{(n+1)}$ are left-most and right-most subintervals of $[0,1)$, with endpoints 0 and 1 respectively (see Figure 1, left). Put

$$
\Delta_{j}^{(n)}:=R_{\alpha}^{j} \Delta_{0}^{(n)} .
$$

Denote by $\lambda^{(n)}$ the length of $\Delta^{(n)}$. Clearly $\lambda^{(n)}$ is also the length of any interval $\Delta_{j}^{(n)}$.

For any $n$, the intervals $\Delta_{j}^{(n)}, 0 \leq j<q_{n+1}$ and $\Delta_{j}^{(n+1)}, 0 \leq j<q_{n}$ are pairwise disjoint and their union is the whole interval $[0,1)$ (see Figure1 left). Denote by $\xi^{(n)}$ the partition of $[0,1)$ into the intervals $\Delta_{j}^{(n)}$ with $0 \leq j<q_{n+1}$ and $\Delta_{j}^{(n+1)}$ with $0 \leq j<q_{n}$. Then $\xi^{(n+1)} \geq \xi^{(n)}$ in the sense of partitions.

Consider the union $\Delta(n):=\Delta^{(n)} \cup \Delta^{(n+1)}$. The set $\Delta(n)$, which, as a subset of $[0,1)$, is the union of two intervals, can be considered $(\bmod 1)$ as a subinterval of the unit circle $S^{1}$, with endpoints on the opposite sides of 0 , i. e. when $n$ is even, $\Delta(n)=\left[-\lambda^{(n+1)}, \lambda^{(n)}\right)$ (see Figure 1, right). Consider the induced map $T^{(n)}$ 
obtained as the first return map of $R_{\alpha}$ on $\Delta(n)$. Then $T^{(n)}$ is an exchange of the two intervals $\Delta^{(n+1)}$ and $\Delta^{(n)}$. More precisely, if $n$ is even, then

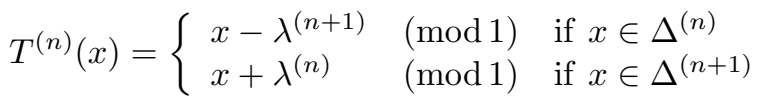

and similarly for odd $n$.

Assume $n$ is even. The intervals $\Delta_{j}^{(n)}$ and $\Delta_{j}^{(n+1)}$ can be represented as floors of two towers, on the top of $\Delta_{0}^{(n)}$ and $\Delta_{0}^{(n+1)}$ respectively, where $j$ increases with the height of the floor in the tower, as in Figure 1 left. Hence the number of floors in the two towers are $q_{n+1}$ and $q_{n}$ respectively. Let us denote the two towers by 1

$$
Z_{l}^{(n)}=\cup_{j=0}^{q_{n+1}-1} \Delta_{j}^{(n)} ; \quad Z_{s}^{(n)}=\cup_{j=0}^{q_{n}-1} \Delta_{j}^{(n+1)} .
$$

Under the action of $R_{\alpha}$ each point not in the last floor (i.e. not in $\Delta_{q_{n+1}-1}^{(n)}$ or $\left.\Delta_{q_{n}-1}^{(n+1)}\right)$ moves vertically upwards to the next floor. The action on the last floor is determined by $T^{(n)}$ : if e.g. $x \in \Delta_{q_{n+1}-1}^{(n)}$ and $x=R_{\alpha}^{q_{n+1}-1} y$ then $R_{\alpha} x=T^{(n)} y$.

1.1.1. Recursive structure of the partitions. Let us also recall how to construct $\xi^{(n)}$ inductively. Given $\xi^{(n)}$, the partition $\xi^{(n+1)}$ is obtained from $\xi^{(n)}$ as follows: the intervals $\Delta_{j}^{(n+1)}, 0 \leq j<q_{n}$ are also elements of the partition $\xi^{(n+1)}$. Each $\Delta_{j}^{(n)}$ is decomposed in $a_{n+2}+1$ subintervals, more precisely in $a_{n+2}$ intervals of length $\lambda^{(n+1)}$ and a reminder, which is $\Delta_{j}^{(n+2)}$ (see for example Figure [3). If $n$ is even, the reminder is the left-most interval of $\Delta_{j}^{(n)}$, while the other intervals, from left to right, are $\Delta_{q_{n}+j+i q_{n+1}}^{(n+1)}$ with $i=0, \ldots, a_{n+2}-1$ (as in Figure 1, left). Hence, we have the following remark.

Remark 1.1. Each pair of intervals of $\xi^{(n)}$ both belonging to the tower $Z_{s}^{(n)}$ are separated by $a_{n+1}$ partition elements belonging to $Z_{l}^{(n)}$.

Given $m<n$, consider $\Delta_{j}^{(m)} \in \xi^{(m)}$. Since $\xi^{(n)}>\xi^{(m)}, \Delta_{j}^{(m)}$ is partitioned into elements of $\xi^{(n)}$. Analyzing the recursive construction of the partitions $\xi^{(n)}$, we have the following.

Remark 1.2. The partition of $\Delta_{j}^{(m)}$ into elements of $\xi^{(n)}$ is completely determined by $\lambda^{(n)}, \lambda^{(n+1)}$ and $a_{n-k+2}, k=0, \ldots, n-m$.

1.2. The renewal-type limit theorem for denominators. The existence of the limiting distribution relies on the following limit theorem. Let $p_{n} / q_{n}$ be the approximants of $\alpha=\left[a_{1}, a_{2}, \ldots\right]$ and $q_{n}=q_{n}(\alpha)$ the corresponding denominators as functions of $\alpha$.

Theorem 3 ( SU08). Given $N>0$, introduce

$$
n(N)=n(N, \alpha)=\min \left\{n \in \mathbb{N} \mid q_{n}>N \text { and } n \text { is even }\right\} .
$$

Fix also an integer $M \geq 0$. Then the ratio $\frac{q_{n(N)}}{N}$ and the entries $a_{n(N)+k}$ for $|k| \leq M$ have a joint limiting distribution, as $N$ tends to infinity, with respect to the uniform distribution on $\alpha$.

\footnotetext{
${ }^{1}$ The subscripts $l$ and $s$ stay for large and small respectively, since the tower $Z_{l}^{(n)}$ is both larger and taller than $Z_{s}^{(n)}$.
} 
Theorem 3 means that for each $M \geq 0$ there exists a probability measure $P_{M}$ on $(1, \infty) \times \mathbb{N}_{+}{ }^{2 M+1}$ such that for all $a, b>1$ and $c_{k} \in \mathbb{N}_{+}$with $|k| \leq M$,

$$
\begin{array}{r}
\lim _{N \rightarrow \infty} \operatorname{Leb}\left\{\alpha: a<\frac{q_{n(N)}(\alpha)}{N}<b, \quad a_{n(N)+k}=c_{k},|k| \leq M\right\}= \\
P_{M}\left((a, b), c_{-M}, \ldots, c_{M}\right) .
\end{array}
$$

Theorem 3 is a slight modification of Theorem 1, SU08. The differences and a sketch on how to modify the proof of Theorem 1 in SU08 to obtain Theorem 3 are pointed out in the Appendix $\mathrm{A} .2$

As a corollary of Theorem 3 , we have the following.

Corollary 1.3. The quantities

$$
\frac{q^{(n(N))}}{q^{(n(N)+1)}}, \quad \frac{\lambda^{(n(N)+1)}}{\lambda^{(n(N))}}, \quad \frac{1}{q^{(n(N))} \lambda^{(n(N)+1)}}, \quad \frac{1}{q^{(n(N)+1)} \lambda^{(n(N))}}
$$

have a limiting distribution as $N$ tends to infinity.

PRoOF. Let us recall that $q_{n}$ and $\lambda^{(n)}$ satisfy the following recurrent relations (see [Khi35] and Sin94] respectively):

$$
q_{n+1}=a_{n+1} q_{n}+q_{n-1}, \quad \lambda^{(n-1)}=a_{n+1} \lambda^{(n)}+\lambda^{(n+1)}, \quad n \geq 1 .
$$

Using them inductively (see Khi35] or [SU08), it is easy to show that

$$
\frac{q_{n}}{q_{n+1}}=\left[a_{n+1}, a_{n}, \ldots, a_{1}\right], \quad \frac{\lambda^{(n+1)}}{\lambda^{(n)}}=\left[a_{n+2}, a_{n+3}, \ldots\right] .
$$

Moreover, reasoning as in [SU08, we also have

$$
\begin{aligned}
& \left|\frac{q_{n}}{q_{n+1}}-\left[a_{n+1}, a_{n}, \ldots, a_{n-K}\right]\right| \leq \frac{1}{2^{\frac{K+1}{2}}} \\
& \left|\frac{\lambda^{(n+1)}}{\lambda^{(n)}}-\left[a_{n+2}, a_{n+3}, \ldots, a_{n+K}\right]\right| \leq \frac{1}{2^{\frac{K-2}{2}}},
\end{aligned}
$$

where the exponential convergence is uniform in $\alpha$. Hence, since by Theorem 3 , for each $K, a_{n(N)}, a_{n(N) \pm 1}, \ldots, a_{n(N) \pm K}$ have a joint limiting distribution as $N$ tends to infinity, $\frac{q_{n(N)}}{q_{n(N)+1}}$ and $\frac{\lambda^{(n(N)+1)}}{\lambda^{(n(N))}}$ also have a limiting distribution.

For the last two quantities, recall, e.g. from [Khi35], that

$$
\lambda^{(n)}=\left|q_{n} \alpha-p_{n}\right|=\frac{1}{q_{n+1}+q_{n} \alpha_{n+1}}, \quad \text { where } \alpha_{n+1}=\mathscr{G}^{n+1} \alpha=\left[a_{n+2}, a_{n+3}, \ldots\right] .
$$

Hence, in particular

$$
\frac{1}{2} \leq \lambda^{(n)} q_{n+1} \leq 1, \quad \lambda^{(n+1)} q_{n} \leq 1
$$

Moreover, since

$$
\frac{1}{q_{n(N)+1} \lambda^{(n(N))}}=1+\frac{q_{n(N)}}{q_{n(N)+1}}\left[a_{n(N)+2}, a_{n(N)+3}, \ldots\right]
$$

the ratio $\frac{1}{q_{n(N)+1} \lambda^{(n(N))}}$ and similarly $\frac{1}{q_{n(N)} \lambda^{(n(N)+1)}}$ have limiting distributions. 


\section{Analysis of a cycle.}

In this section and in 93 , we consider only Birkhoff sums of the function $f_{1}$. Since $f_{2}$ is integrable, Birkhoff sums of $f_{2}$ are easily controlled in $\$ 4$ with the help of Birkhoff ergodic theorem.

We first investigate in this section a special type of Birkhoff sum, which is used in 93 as a building block to decompose any other Birkhoff sum. Assume that $x \in \Delta^{(n)}$ and $q=q^{(n+1)}$ if $n$ is even or $q=q^{(n-1)}$ if $n$ is odd and consider the Birkhoff sum $S_{q}(\alpha, x)$. We call the orbit segment $\left\{R_{\alpha}^{i} x, i=0, \ldots, q-1\right\}$ a cycle and $S_{q}(\alpha, x)$ is a sum over a cycle. We remark that all points of a cycle are contained in the same tower and there is exactly one point in each floor of the tower; for this reason, we sometimes refer to $S_{q}(\alpha, x)$ as a sum over a tower (see also [Ulc07]). In section $\$ 3$ we will refer to $n$ as the order of the cycle.

To simplify the analysis, we assume in what follows that $n$ is even and consider only the partitions $\xi^{(n)}$ with $n$ even and their cycles. The following proposition shows that the value of a sum over a cycle is determined essentially by the closest point to the endpoint.

Proposition 2.1. Let $S_{q}(\alpha, x)$ be a sum along a cycle, $q=q_{n+1}$ if $x \in \Delta^{(n)}$ or $q=q_{n}$ if $x \in \Delta^{(n+1)}$. For each $\epsilon>0$ there exist $K=K(\epsilon)$ and functions $g_{n}^{\epsilon}(\alpha, x, q), n \in \mathbb{N}$, which depend only on the following quantities

$g_{n}^{\epsilon}(\alpha, x, q)= \begin{cases}g_{n}^{\epsilon}\left(\frac{x}{\lambda^{(n)}}, \frac{\lambda^{(n+1)}}{\lambda^{(n)}}, \frac{1}{q_{n+1} \lambda^{(n)}}, a_{n+2}, a_{n+1}, \ldots, a_{n-K}\right) & \text { if } x \in \Delta^{(n)} \\ g_{n}^{\epsilon}\left(\frac{x}{\lambda^{(n+1)}}, \frac{\lambda^{(n+1)}}{\lambda^{(n)}}, \frac{1}{q_{n} \lambda^{(n+1)}}, a_{n+2}, a_{n+1}, \ldots, a_{n-K}\right) & \text { if } x \in \Delta^{(n+1)}\end{cases}$

and such that, letting $q=q^{(n+1)}$ if $x \in \Delta^{(n)}$ or $q=q_{n}$ if $x \in \Delta^{(n+1)}$, we have

$$
\left|\frac{1}{q} S_{q}(\alpha, x)-g_{n}^{\epsilon}(\alpha, x, q)\right| \leq \epsilon .
$$

The proof of Proposition 2.1 is given in 92.2 The key ingredient which allows to reduce the sum over a cycle to finitely many terms (and hence to an expression given by $g_{\epsilon}$ depending on the above variables) is that there are cancellations between the two sides, positive and negative, of the singularity. The cancellations occur because the sequence of closest points to 0 is given by a rigid translate of the sequence of closest points to 1 (see Corollary 2.5). In order to prove this fact, we first show, in 2.1 that the partitions $\xi^{(n)}$ have a property of almost symmetry (see Lemma 2.3, in $\sqrt{2.11}$.

2.1. Almost symmetry of the partitions. Consider the partition $\xi^{(n)}$ and let $z_{i}^{(n)}$, for $i=0, \ldots, q_{n+1}-1$ denote the middle points of the intervals $\Delta_{j}^{(n)}$, $0 \leq j<q_{n+1}$, rearranged in increasing order, so that $z_{0}^{(n)}<z_{1}^{(n)}<\cdots<z_{q_{n+1}-1}^{(n)}$ and similarly let $z_{i}^{(n+1)}$, for $i=0, \ldots, q_{n}-1$ be the middle points of the intervals $\Delta_{j}^{(n+1)}, 0 \leq j<q_{n}$ rearranged in increasing order (see Figure 2).

Since we are interested in comparing the functions $\frac{1}{x}$ and $\frac{1}{1-x}$ evaluated along orbits segments which contain a point inside each of these intervals, we want to understand what happens to the middle points under the reflection $x \mapsto \sigma(x):=$ $(1-x)$. 


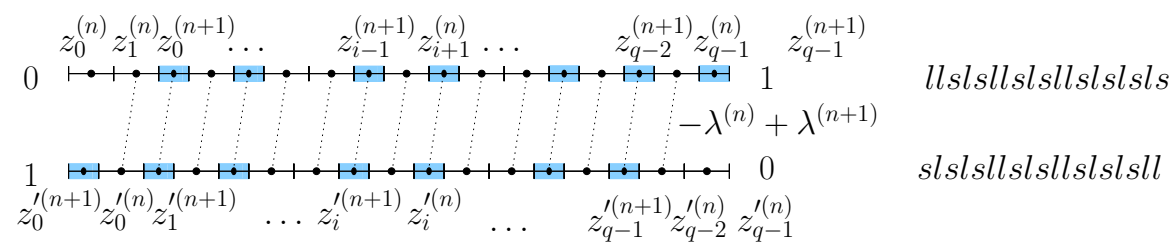

Figure 2. An example of the relations (2.3, 2.4) between $z_{i}^{(n)}$, $z_{i}^{(n+1)}$ and $z_{i}^{\prime(n)}, z_{i}^{\prime(n+1)}$.

Consider the set of reflected points $\left\{1-z_{i}^{(n)}\right.$, for $\left.i=0, \ldots, q_{n+1}-1\right\}$ and let $z_{i}^{\prime(n)}$, for $i=0, \ldots, q_{n+1}-1$ denote its elements rearranged in increasing order. Similarly, let $z_{i}^{\prime(n+1)}$, for $i=0, \ldots, q_{n}-1$ be the monotonical rearrangements of the points $\left\{1-z_{i}^{(n+1)}\right.$, for $\left.i=0, \ldots, q_{n}-1\right\}$.

Lemma 2.2. Let $n$ be even. The two sequences given by the points $z_{i}^{(n)}$ and the points $z_{i}^{(n+1)}$ respectively, excluding the closest point to 0 , i.e. $z_{0}^{(n)}$, and the closest point to 1 , i.e. $z_{q_{n}-1}^{(n+1)}$, are rigid translates of each other, i.e. they satisfy:

$$
\begin{array}{rlr}
z_{i}^{\prime(n)} & =z_{i+1}^{(n)}+\lambda^{(n+1)}-\lambda^{(n)} ; & i=0, \ldots, q_{n+1}-2 \\
z_{i}^{\prime(n+1)} & =z_{i-1}^{(n+1)}+\lambda^{(n+1)}-\lambda^{(n)}, & i=1, \ldots, q_{n}-1 .
\end{array}
$$

The restriction on the parity simplify the number of cases in the statement, but similar properties could be proved for $n$ odd.

Lemma 2.2 will follow as a corollary of an almost-symmetry property of the partitions $\xi^{(n)}$ (Lemma 2.3 below). Let us consider the following coding of the partitions $\xi^{(n)}$. The unit interval $[0,1)$ is decomposed into $q_{n}+q_{n+1}$ subintervals which are elements of the partition $\xi^{(n)}$ and either belong to $Z_{l}^{(n)}$ (i.e. are of the form $\Delta_{j}^{(n)}$ for some $0 \leq j<q_{n+1}$ ) or to $Z_{s}^{(n)}$ (i.e. are of the form $\Delta_{j}^{(n+1)}$ for some $0 \leq j<q_{n}$ ). We will call them intervals of type $l$ and type $s$ respectively (large or short). Let $\underline{\omega}^{(n)}=\omega_{1}^{(n)} \cdots \omega_{q_{n}+q_{n+1}}^{(n)}$ be a string of letters $l$ and $s$, where $\omega_{i}^{(n)}=l$ or $\omega_{i}^{(n)}=s$ according to the type of the $i^{t h}$ interval of $\xi^{(n)}$ (where intervals of the partition are ordered from left to right in $[0,1)$ ). For example, the string coding the partition $\xi^{(n)}$ in Figure 2 (which is the same that appears also in Figure 1) is $\underline{\omega}^{(n)}=$ llslsllslsllslslsls.

Let $\underline{\omega}^{\prime(n)}=\omega_{q_{n+1}}^{\prime(n)}, \ldots, \omega_{1}^{\prime(n)}$ be the reflected string, which encodes the type of intervals after the reflection $x \mapsto 1-x$. Then, the following almost-symmetry property is satisfied by the partitions $\xi^{(n)}$.

Lemma 2.3 (almost symmetry of $\xi^{(n)}$ ). For all $n$, all the letters of the strings $\underline{\omega}^{(n)}$ and $\underline{\omega}^{(n)}$ coincide with the exception of the first and last, i.e.

$$
\omega_{i}^{\prime(n)}=\omega_{i}^{(n)} \quad \text { for all } \quad 2 \leq i \leq q_{n}+q_{n+1}-1 .
$$

More precisely,

$$
\begin{aligned}
& \text { if } n \text { is even, } \underline{\omega}^{(n)}=\left(l \omega_{2}^{(n)} \cdots \omega_{q_{n}+q_{n+1}-1}^{(n)} s\right) \text { and } \underline{\omega}^{\prime(n)}=\left(s \omega_{2}^{(n)} \cdots \omega_{q_{n}+q_{n+1}-1}^{(n)} l\right) ; \\
& \text { if } n \text { is odd, } \underline{\omega}^{(n)}=\left(s \omega_{2}^{(n)} \cdots \omega_{q_{n}+q_{n+1}-1}^{(n)} l\right) \text { and } \underline{\omega}^{\prime(n)}=\left(l \omega_{2}^{(n)} \cdots \omega_{q_{n}+q_{n+1}-1}^{(n)} s\right) .
\end{aligned}
$$




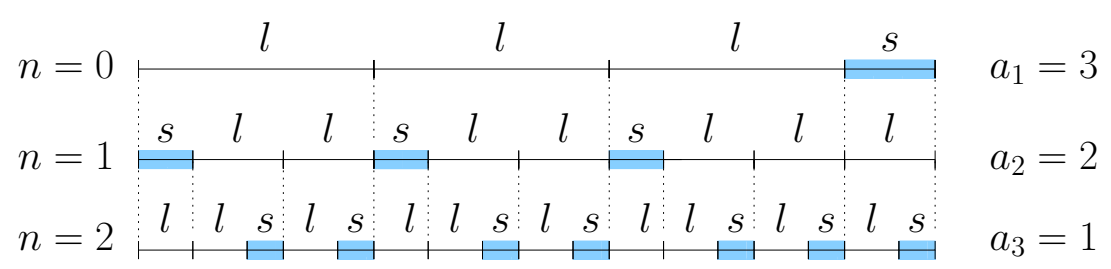

Figure 3. An example of partitions $\xi^{(0)}, \xi^{(1)}, \xi^{(1)}$ (where $a_{1}=3$, $\left.a_{2}=2, a_{3}=1\right)$.

Moreover, for $n \geq 4, \omega_{2}^{(n)}=\omega_{2}^{\prime(n)}=\omega_{q_{n}+q_{n+1}-1}^{(n)}=\omega_{q_{n}+q_{n+1}-1}^{(n)}=l$.

Proof. The proof proceeds by induction on $n$. For $n=0, \underline{\omega}^{(0)}=(l l \cdots l s)$ where the number of occurrences of $l$ is given by $a_{1}$. Hence, $\underline{\omega}^{\prime(0)}=(\operatorname{sll} \cdots l)$ and there is nothing to prove. Assume that the almost-symmetry is proved for $\xi^{(n)}\left(\omega_{i}^{\prime}=\omega_{i}\right.$ for all $\left.2 \leq i \leq q_{n}+q_{n+1}-1\right)$ and $n$ is even. As it can be seen easily analyzing the recursive construction of $\xi^{(n)}$ in $\$ 1.1$, the new string $\underline{\omega}^{(n+1)}$ is obtained from $\underline{\omega}^{(n)}$ by substituting each letter $s$ with $l$ (since $\lambda^{(n+1)}$ which was the shortest length in $\xi^{(n)}$ is now the longest one in $\left.\xi^{(n+1)}\right)$ and substituting each letter $l$ with $s l l \ldots l$ where the number of occurrences $l$ is given by $a_{n+1}$, see for example Figure 3 .

To verify the desired identities on the letters in $\underline{\omega}^{(n+1)}$ and $\underline{\omega}^{\prime(n+1)}$ it is enough to verify that the letters $s$ occur in the same positions (with the exception the first and last letter of the string). Let $l(i)$ denote the number of letters $l$ among $\omega_{j}^{(n)}$ with $0 \leq j<i$ (i.e. the cardinality of $\omega_{j}^{(n)}=l$ with $0 \leq j<i$ ). Since all $s$ in $\underline{\omega}^{(n)}$ become $l$, the only $s$ in the string $\underline{\omega}^{(n+1)}$ appear inside each block sll $\ldots l$. Moreover, each occurrence of $l$ in $\underline{\omega}^{(n)}$ generates a string of length $a_{n+1}+1$ in $\underline{\omega}^{(n+1)}$. Hence $\omega_{j}^{(n+1)}=s$ iff $\omega_{i}^{(n)}=l$ and $j=i+a_{n+1} l(i)$ (for $1 \leq i \leq q_{n}+q_{n+1}$ ).

Similarly the string $\underline{\omega}^{\prime(n+1)}$ is obtained from $\underline{\omega}^{\prime(n)}$ by substituting $s$ with $l$ and substituting each symbol $l$ with $l l \ldots l s\left(a_{n+1}\right.$ copies of $\left.l\right)$. If $l^{\prime}(i)$ denote the number of letters $l$ (i.e. $\omega_{j}^{\prime(n)}=l$ ) among $\omega_{j}^{\prime(n)}$ with $0 \leq j<i$, then ${\omega^{\prime}}_{j^{\prime}}^{(n)}=s$ iff $\omega_{i}^{(n)}=l$ and $j^{\prime}=i+a_{n+1}\left(l^{\prime}(i)+1\right.$ ) (for $\left.i=1, \ldots, q_{n}+q_{n+1}\right)$. By the inductive assumption, since $\omega_{i}^{(n)}$ and $\omega_{i}^{\prime(n)}$ coincide for all $i \neq 1, i \neq q_{n}+q_{n+1}$ but $\omega_{1}^{\prime(n)}=s$, we have $l^{\prime}(i)=l(i)-1$. Hence, for $2 \leq i \leq q_{n-1}+q_{n-2}-1$, we have $\omega_{i}^{(n)}=l$ iff $\omega_{i}^{\prime(n)}=l$ and $j^{\prime}=i+a_{n+1}\left(l^{\prime}(i)+1\right)=i+a_{n+1} l(i)=j$, which implies, as we wanted, that ${\omega^{\prime}}_{j^{\prime}}^{(n+1)}=s$ iff $\omega_{j}^{(n+1)}=s$. The proof for odd $n$ is analogous.

From the definition of $\Delta^{(n)}$ we have immediately that $\omega_{1}^{(n)}=l, \omega_{q_{n-1}+q_{n-2}}^{(n)}=s$ for $n$ even and $\omega_{1}^{(n)}=s, \omega_{q_{n-1}+q_{n-2}}^{(n)}=l$ for $n$ odd and the last equalities follow from the (2.5) and the fact that two $s$ are never nearby.

Proof of Lemma 2.2. Assume $n$ is even. The points $z_{i}^{(n)}$ and ${z^{\prime}}_{i}^{(n)}$ for $0 \leq$ $i<q_{n+1}$ are middle points of intervals of type $l$ respectively before and after the reflection. Let us first prove (2.3) for $i=0$. The first interval of the partition $\xi^{(n)}$ is of type $l$ and hence contains $z_{0}^{(n)}$, while ${z^{\prime}}_{0}^{(n)}$ belongs to the second interval after the reflection, since $\omega_{0}^{\prime(n)}=s$. Unless the string has the length 2 and is $l s$ (in which case there is nothing to prove), by Lemma 2.3 also $\omega_{1}^{(n)}=l$, so the first two 


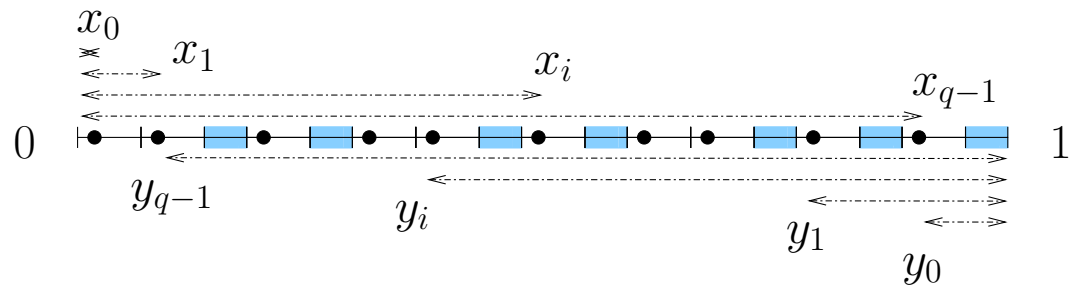

Figure 4. The distances $x_{i}$ and $y_{i}, 0 \leq i<q$, from 0 and 1 respectively ( $n$ even).

intervals are both of type $l$ and we have ${z^{\prime}}^{(n)}=z_{1}^{(n)}-\lambda^{(n)}+\lambda^{(n+1)}$. Moreover, since by Lemma 2.3 , the strings $\underline{\omega}^{(n)}$ and $\underline{\omega}^{\prime(n)}$ coincide after the first element, also $z_{i}^{\prime(n)}=z_{i+1}^{(n)}-\lambda^{(n)}+\lambda^{(n+1)}$ for all $i=0, \ldots, q_{n+1}-2$ (see Figure 2).

Similarly, the points $z_{i}^{(n+1)}$ and ${z^{\prime}}^{(n+1)}$ for $0 \leq i<q_{n}$ are middle points of intervals of type $s$. In this case, ${z^{\prime}}_{0}^{(n+1)}$ belongs to the first interval after the reflection $\left(\omega_{0}^{\prime(n)}=s\right)$ and has to be kept aside, while $z_{0}^{(n+1)}$ and ${z^{\prime}}_{1}^{(n+1)}$ belong respectively to the $\left(a_{n+2}+1\right)^{t h}$ interval before reflection and to the $\left(a_{n+2}+1\right)^{t h}$ after reflection. Since the strings are $\omega_{0}^{(n)} \omega_{1}^{(n)} \cdots \omega_{a_{n+1}}^{(n)}=l l \cdots l s$ and ${\omega^{\prime}}_{0}^{(n)} \omega_{1}^{(n)} \cdots \omega_{a_{n+1}+2}^{(n)}=$ $s l \cdots l s$ respectively, $z_{1}^{\prime(n+1)}=z_{0}^{(n+1)}-\left(a_{n+2}+1\right) \lambda^{(n)}+a_{n+2} \lambda^{(n)}+\lambda^{(n+1)}=$ $z_{0}^{(n+1)}-\lambda^{(n)}+\lambda^{(n+1)}$ and, again by Lemma 2.3, since the strings then coincide, also ${z^{\prime}}_{i}^{(n+1)}=z_{i-1}^{(n)}-\lambda^{(n)}+\lambda^{(n+1)}$ for all $i=1, \ldots, q_{n}-1$ (see again Figure 2).

2.2. Cancellations. Let $n$ be even, $x \in \Delta(n)$ and let $q=q_{n}$ or $q_{n+1}$ according to whether $x \in \Delta^{(n+1)}$ or $x \in \Delta^{(n)}$. Consider the orbit cycle $\left\{R_{\alpha}^{i} x: i=0, \ldots, q-\right.$ $1\}$, which is an orbit along a tower of $\xi^{(n)}$. Let us rename the points of $\left\{R_{\alpha}^{i} x\right\}_{i=0}^{q-1}$ in increasing order, so that

$$
0<x_{0}<x_{1}<\cdots<x_{q-1}<1, \quad \bigcup_{i=0}^{q-1}\left\{R_{\alpha}^{i} x\right\}=\bigcup_{i=0}^{q-1}\left\{x_{i}\right\} .
$$

Similarly rearrange in increasing order distances from 1, i. e. the elements of $\{1-$ $\left.R_{\alpha}^{i} x\right\}_{i=0}^{q-1}$, renaming them by

$$
0<y_{0}<y_{1}<\cdots<y_{q-1}<1, \quad \bigcup_{i=0}^{q-1}\left\{1-R_{\alpha}^{i} x\right\}=\bigcup_{i=0}^{q-1}\left\{y_{i}\right\} .
$$

From the structure of the partitions described in the second part of Lemma 2.3 one can easily check the following (see also Figure 5).

Remark 2.4. If $x \in \Delta^{(n)}, x=x_{0}$ and $y_{0}=\lambda^{(n+1)}+\lambda^{(n)}-x_{0}$, while if $x \in \Delta^{(n+1)}$, $y_{0}=1-x$ and $x_{0}=\lambda^{(n)} a_{n+1}+\lambda^{(n+1)}-y_{0}$.

For the other points, from the partition almost-symmetry expressed by Lemma 2.2. we have the following (see an illustration in Figure 4).

Corollary 2.5. For all $1 \leq j \leq q$

$$
\begin{aligned}
& y_{i}-x_{i+1}=\lambda^{(n+1)}-2 x_{0}, \quad i=0, \ldots, q_{n+1}-2, \quad \text { if } x \in \Delta^{(n)} \\
& y_{i}-x_{i-1}=2 y_{0}-\lambda^{(n)}, \quad i=1, \ldots, q_{n}-1 \quad \text { if } x \in \Delta^{(n+1)}
\end{aligned}
$$




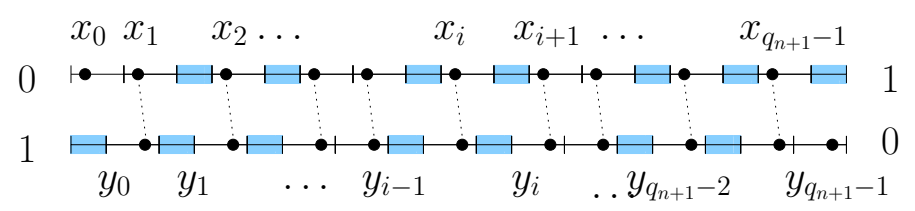

(a) $x \in \Delta^{(n)}, q=q_{n+1}$

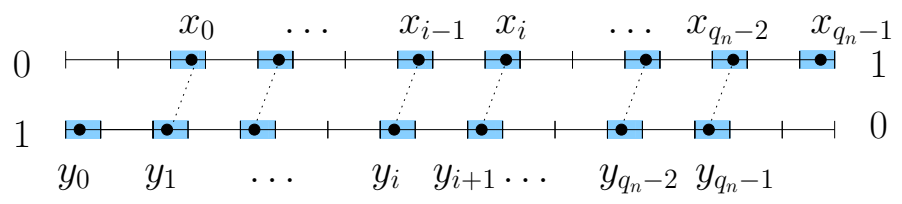

(b) $x \in \Delta^{(n+1)}, q=q_{n}$

Figure 5. An example of the relations (2.6, 2.7) between $x_{i}$ and $y_{i}$.

Proof. Assume that $x \in \Delta^{(n)}$ (see Figure 5(a) $)$. Since, for some $0 \leq k<q_{n+1}$, $x_{i}$ and $z_{i}^{(n)}$ both belong to the same $\Delta_{k}^{(n)}$, which is a rigid translate of $\Delta_{0}^{(n)}$, we have $x_{i}-z_{i}^{(n)}=x_{0}-z_{0}^{(n)}=x_{0}-\lambda^{(n)} / 2$. Similarly, both $y_{j}$ and ${z^{\prime}}_{j}^{(n)}$ belong to the same $\Delta_{k^{\prime}}^{(n)}$, which is a rigid translate and a reflection of $\Delta_{0}^{(n)}$, hence $y_{j}-z_{j}^{(n)}=\lambda^{(n)} / 2-x_{0}$. Thus, $y_{j}-x_{i}={z^{\prime}}_{j}^{(n)}-z_{i}^{(n)}+\lambda^{(n)}-2 x_{0}$. Using this relation, (2.6) follows from (2.3) in Lemma 2.2. The argument to prove (2.7) when $x \in \Delta^{(n+1)}$ is analogous (see Figure 5(b) and reduces to (2.4) in Lemma 2.2

We remark that the points of $\left\{R_{\alpha}^{i} x, i=0, \ldots, q-1\right\}$ belong to different floors of the tower of the partition $\xi^{(n)}$ and are in the same relative position inside them. Hence, we have the following.

Remark 2.6. The minimum distance $\min _{i \neq j}\left|x_{i}-x_{j}\right|$ is bounded below by $\lambda^{(n)}$. In particular, $x_{j} \geq j \lambda^{(n)}$ and similarly $y_{j} \geq j \lambda^{(n)}$ for $1 \leq j \leq q_{n+1}-1$.

Moreover, by Remark 1.1. two floors of type $s$ have always $a_{n+1}$ floor of type $l$ in between them and since, if $x_{0} \in \Delta^{(n+1)}$, all points $x_{i}$ with $0 \leq i \leq q_{n}-1$, belong to different floors of type $s$, we also have the following.

Remark 2.7. If $x_{0} \in \Delta^{(n+1)}, x_{j} \geq j a_{n+1} \lambda^{(n)} \geq j \frac{a_{n+1}}{a_{n+1}+1} \lambda^{(n-1)} \geq \frac{j}{2} \lambda^{(n-1)}$ and $y_{j} \geq \frac{j}{2} \lambda^{(n-1)}$, for $1 \leq j \leq q_{n}-1$.

Applying Corollary 2.5] and Remark 2.6, we can control cancellations through a converging series and prove the following Lemma, which shows that the main contribution to the sum along a cycle is determined by the closest visits to 0 and 1 .

Lemma 2.8. For each $\epsilon>0$, there exists $k(\epsilon)$ such that for all $k \geq k(\epsilon)$, if $S_{q}\left(\alpha, x, f_{1}\right)$ is a sum along a cycle of order $n$,

$$
\left|\frac{1}{q} S_{q}\left(\alpha, x, f_{1}\right)-\frac{1}{q} \sum_{i=0}^{k}\left(\frac{1}{x_{i}}-\frac{1}{y_{i}}\right)\right| \leq \epsilon .
$$


Proof. Using the new labeling of the orbit points, introduced at the beginning of $\Varangle 2.2$, we have

$$
S_{q}(\alpha, x)=\sum_{i=0}^{q-1}\left(\frac{1}{R_{\alpha}^{i} x}-\frac{1}{1-R_{\alpha}^{i} x}\right)=\sum_{i=0}^{q-1}\left(\frac{1}{x_{i}}-\frac{1}{y_{i}}\right) .
$$

Let us apply Corollary 2.5 to control $\left|y_{i}-x_{i+1}\right|$ or $\left|x_{i}-y_{i+1}\right|$ respectively. In the case $x \in \Delta^{(n)}$, rearranging the terms of the summation to use (2.6), we get

$$
\begin{aligned}
S_{q}(\alpha, x)-\sum_{i=0}^{k}\left(\frac{1}{x_{i}}-\frac{1}{y_{i}}\right) & =\sum_{i=k}^{q-2}\left(\frac{1}{x_{i+1}}-\frac{1}{y_{i}}\right)+\frac{1}{y_{k}}-\frac{1}{y_{q-1}} \\
& =\sum_{i=k}^{q-2} \frac{\lambda^{(n+1)}-2 x_{0}}{y_{i} x_{i+1}}+\frac{1}{y_{k}}-\frac{1}{y_{q-1}} .
\end{aligned}
$$

From (2.9), using Remark 2.6 which gives $x_{i}, y_{i} \geq i \lambda^{(n)}$, we have, as long as $k \geq 1$,

$$
\left|\frac{1}{q} S_{q}(\alpha, x)-\frac{1}{q} \sum_{i=0}^{k}\left(\frac{1}{x_{i}}-\frac{1}{y_{i}}\right)\right| \leq\left|\frac{1}{q \lambda^{(n)}} \sum_{i=k}^{q-2} \frac{\frac{\lambda^{(n+1)}}{\lambda^{(n)}}-\frac{2 x_{0}}{\lambda^{(n)}}}{i^{2}}\right|+\frac{1}{q y_{k}} .
$$

The second term in the RHS, by Remark 2.6 and (1.4), is bounded by $\frac{1}{q y_{k}} \leq$ $\left(k q_{n+1} \lambda^{(n)}\right)^{-1} \leq \frac{2}{k}$ and hence by $\epsilon / 2$ if $k \geq k(\epsilon)$ where $k(\epsilon)$ is large enough. Moreover, since $\left(q \lambda^{(n)}\right)^{-1}=\left(q_{n+1} \lambda^{(n)}\right)^{-1} \leq 2$ by (1.4),$\lambda^{(n+1)} / \lambda^{(n)} \leq 1$ and $x_{0} / \lambda^{(n)} \leq 1$, the first term in the RHS is bounded by the remainder of a converging series. Hence, we can choose $k(\epsilon)$ large enough so that also $6 \sum_{k(\epsilon)}^{\infty} i^{-2}<\epsilon / 2$ and this concludes the proof in the case $x \in \Delta^{(n)}$. In the case $x \in \Delta^{(n+1)}$ and $q=q_{n}$, in an analogous way we get

$$
S_{q}(\alpha, x)=\sum_{i=0}^{k}\left(\frac{1}{x_{i}}-\frac{1}{y_{i}}\right)+\sum_{i=k+1}^{q-1}\left(\frac{1}{x_{i-1}}-\frac{1}{y_{i}}\right)-\frac{1}{x_{k}}+\frac{1}{x_{q-1}}
$$

and this time by (2.7) and Remark 2.7 and also (1.4) and $y_{0} \leq \lambda^{(n+1)}$, we have

$$
\left|\frac{1}{q} \sum_{i=k+1}^{q-1}\left(\frac{1}{x_{i-1}}-\frac{1}{y_{i}}\right)\right| \leq\left|\frac{4}{q} \sum_{i=k+1}^{q-1} \frac{2 y_{0}-\lambda^{(n)}}{\left(\lambda^{(n-1)}\right)^{2} i^{2}}\right| \leq \frac{12}{q_{n} \lambda^{(n-1)}} \sum_{i=k+1}^{q-1} \frac{\lambda^{(n)}}{\lambda^{(n-1)}} \leq \sum_{i=k+1}^{q-1} \frac{24}{i^{2}} \text {. }
$$

Moreover, using again Remark 2.7, one has $\frac{1}{q}\left|\frac{1}{x_{q-1}}-\frac{1}{x_{k}}\right| \leq \frac{1}{q x_{k}} \leq \frac{2}{k q_{n} \lambda^{(n-1)}} \leq \frac{4}{k}$ so by choosing $k(\epsilon)$ large enough this concludes the proof also in this second case.

Corollary 2.9. There exists $M>0$ such that for all sums $S_{q}(\alpha, x)$ along a cycle we get

$$
\left|\frac{1}{q} S_{q}(\alpha, x)\right| \leq \max \left\{\frac{1}{q x_{0}}, \frac{1}{q y_{0}}\right\}+M .
$$

Proof. It follows from the estimates in the proof of Lemma 2.8 for $k=0$, if we take $M=24 \sum_{i=1}^{\infty} i^{-2}$.

We are now ready to prove Proposition 2.1 . 
Proof of Proposition 2.1, Given $\epsilon>0$, choose $k(\epsilon)$ so that Lemma 2.8 holds. The value of $S_{q}(\alpha, x) / q$ is hence determined up to $\epsilon$ by the contribution from any number $k \geq k(\epsilon)$ of closest points to 0 and 1 . Let us show that the positions of $x_{i}$ and $y_{i}$ with $0 \leq i \leq k(\epsilon)$ are determined by $x$ and $a_{n+2-k}$ with $0 \leq k \leq K(\epsilon)$ for some $K(\epsilon)$. Choose $K=K(\epsilon)$ so that $2^{(K(\epsilon)-2) / 2}>k(\epsilon)$. Hence, since the elements of $\xi^{(n)}$ of a fixed type contained inside $\Delta^{(n-K)}$ are at least 2 $2^{\frac{K-1}{2}}$, we have that

$$
\begin{aligned}
& \left\{x_{0}, x_{1}, \ldots, x_{k(\epsilon)}\right\} \subset \Delta^{(n-K(\epsilon))}, \\
& \left\{y_{0}, y_{1}, \ldots, y_{k(\epsilon)}\right\} \subset \Delta^{(n-K(\epsilon)+1)}=\left[1-\lambda^{(n-K(\epsilon)+1)}\right) .
\end{aligned}
$$

Let $q g_{n}^{\epsilon}$ be the sum over all the points $y_{i}, i=0, \ldots, k-1$, which are contained in $\Delta^{(n-K(\epsilon)+1)}$ (here $k \geq k(\epsilon)$ denotes their cardinality) and over the corresponding points $x_{i}, i=0, \ldots, k$, which are all contained in $\Delta^{(n-K(\epsilon))}$. Explicitly, if $x \in \Delta^{(n)}$ (and hence $x=x_{0}$ and $q=q_{n+1}$ )

$g_{n}^{\epsilon}(x, \alpha, q)=\frac{1}{q}\left(\frac{1}{x_{0}}+\sum_{i=0}^{k-1} \frac{\lambda^{(n+1)}-2 x_{0}}{y_{i} x_{i+1}}\right)=\frac{1}{q_{n+1} \lambda^{(n)}}\left(\frac{1}{\frac{x_{0}}{\lambda^{(n)}}}+\sum_{i=0}^{k-1} \frac{\frac{\lambda^{(n+1)}}{\lambda^{(n)}}-\frac{2 x_{0}}{\lambda^{(n)}}}{\frac{y_{i}}{\lambda^{(n)}} \frac{x_{i}+1}{\lambda^{(n)}}}\right)$.

and a similar expression can be written for $x \in \Delta^{(n+1)}$.

We remark that the points $x_{i}$ with $0 \leq i \leq k$ belong to floors of the tower $Z_{l}^{(n)}$ of the partition $\xi^{(n)}$ which are contained in $\Delta^{(n-K(\epsilon))}$ and are determined once $x_{0}$ and the relative position of these floors is given. Similarly, $y_{i}$ with $0 \leq i \leq k$ belong to the floors of the tower $Z_{0}^{(n+1)}$ of the partition $\xi^{(n)}$ which are contained in $\Delta^{(n-K(\epsilon))}$ and are determined once $y_{0}$ and the relative position of the floors are given.

By Remark 2.4 $x_{0}$ and $y_{0}$ can be expressed through $x, \lambda^{(n)}, \lambda^{(n+1)}$ and $a_{n+1}$. Moreover, by Remark 1.2, the lengths $\lambda^{(n)}$ and $\lambda^{(n+1)}$ and the entries $a_{n+2-k}$ with $k \leq K(\epsilon)$ determine the sequence of floors of $\xi^{(n)}$ both inside $\Delta^{(n-K(\epsilon))}$ and inside $\Delta^{(n-K(\epsilon)+1)}$. Dividing all quantities by $\lambda^{(n)}$ if $x \in \Delta^{(n)}$ (or by $\lambda^{(n+1)}$ if $x \in \Delta^{(n+1)}$ ), the ratios $x_{i} / \lambda^{(n)}$ and $y_{i} / \lambda^{(n)}$ (or $x_{i} / \lambda^{(n+1)}$ and $\left.y_{i} / \lambda^{(n+1)}\right)$, which are the quantities through which $g_{n}^{\epsilon}$ is expressed in (2.11), are determined by $x_{0} / \lambda^{(n)}=x / \lambda^{(n)}$ (or $\left.y_{0} / \lambda^{(n+1)}=x / \lambda^{(n+1)}\right), \lambda^{(n+1)} / \lambda^{(n)}$ and the entries $a_{n+2-k}$ with $0 \leq k \leq K(\epsilon)$.

Hence we have shown that $g_{n}^{\epsilon}$ can be expressed through the quantities in (2.1). The relation (2.2) follows immediately from Lemma 2.8

\section{General Birkhoff sums.}

In this section we consider general Birkhoff sums and prove the following.

Proposition 3.1. For each $\epsilon>0, \delta>0$, there exists a function $G^{\epsilon, \delta}=G^{\epsilon, \delta}(x, \alpha, N)$, such that for all sufficiently large $N$,

$$
\operatorname{Leb}\left\{(x, \alpha):\left|\frac{1}{N} S_{N}(\alpha, x)-G^{\epsilon, \delta}(x, \alpha, N)\right| \geq \epsilon\right\} \leq \delta
$$

\footnotetext{
${ }^{2}$ This estimate can be obtained from the recursive relations between $\xi^{(n)}$ and $\xi^{(n+1)}$, see 1.1 and the lower bound for the growth of Fibonacci numbers.
} 
and there exists $K_{1}=K_{1}(\epsilon)$ such that any $G^{\epsilon, \delta}(x, \alpha, N)$ can be expressed as a function of the following quantities:

$$
\begin{aligned}
& G^{\epsilon, \delta}\left(\frac{q_{n}}{N}, \frac{d(x)}{\lambda^{(n-2)}}, \frac{h(x)}{q_{n-1}}, \frac{\lambda^{(n-1)}}{\lambda^{(n-2)}}, \frac{q_{n-2}}{q_{n-1}}, \frac{1}{q_{n-1} \lambda^{(n-2)}}, a_{n}, \ldots, a_{n \pm K_{1}}\right), \text { if } x \in Z_{l}^{(n-2)} \\
& G^{\epsilon, \delta}\left(\frac{q_{n}}{N}, \frac{d(x)}{\lambda^{(n-1)}}, \frac{h(x)}{q_{n-2}}, \frac{\lambda^{(n-1)}}{\lambda^{(n-2)}}, \frac{q_{n-2}}{q_{n-1}}, \frac{1}{q_{n-2} \lambda^{(n-1)}}, a_{n}, \ldots, a_{n \pm K_{1}}\right), \text { if } x \in Z_{s}^{(n-2)}
\end{aligned}
$$$$
\text { where } n:=n(\alpha, N) \text { is as in (1.1) and where } d(x)=d_{n-2}(x) \text { and } h(x)=h_{n-2}(x)
$$
are defined as follows 3

- if $x \in \Delta_{j}^{(n-2)} \subset Z_{l}^{(n-2)}, d(x) \in \Delta_{0}^{(n-2)}$ is such that $R_{\alpha}^{j} d(x)=x$ and $h(x)=q_{n-1}-j$, for some $0 \leq j<q_{n-1}$;

- if $x \in \Delta_{j}^{(n-1)} \subset Z_{s}^{(n-2)}$, then $d(x) \in \Delta_{0}^{(n-1)}$ is such that $R_{\alpha}^{j}(1-d(x))=x$ and $h(x)=q_{n-2}-j$, for some $0 \leq j<q_{n-2}$.

As a corollary of this Proposition, we prove that $G^{\epsilon, \delta}(x, \alpha, N)$ has a joint limiting distribution in $(x, \alpha)$ as $N$ tends to infinity. Indeed, all the quantities through which $G^{\epsilon, \delta}$ is expressed in (3.2) have limiting distributions as $N$ tends to infinity (some of them were considered in $\$ 1.2$, for the other ones see \$4, Lemma 4.1) and, together with the continuity properties of $G^{\epsilon, \delta}(x, \alpha, N)$ (see Lemma 4.3), this implies that $G^{\epsilon, \delta}$ has a limiting distribution.

In order to prove Proposition 3.1 (in \$3.2), we first show how to decompose $S_{N}(\alpha, x)$ into Birkhoff sums along cycles, see 3.1 Then, in $\$ 3.2$ we show that, neglecting a set of $(\alpha, x)$ of small measure, we can reduce the decomposition to finitely many cycles. Hence the function $G^{\epsilon, \delta}$ is defined combining finitely many functions $g_{n}^{\epsilon}$ constructed in Proposition 2.1 to approximate sums along cycles.

3.1. Decomposition into cycles. Fix $N$ and $\alpha$ and let $n=n(N, \alpha)$ be the unique even $n \in \mathbb{N}$ such that $q_{n-2}<N<q_{n}$. The dependence on $N$ and $\alpha$ will be omitted in this section since $N$ and $\alpha$ are fixed throughout. Consider the partition $\xi^{(n-2)}$. We will decompose the orbit $\left\{R_{\alpha}^{i} x, 0 \leq i<N\right\}$ into cycles and $n-2$ will be the biggest order of the cycles involved in the decomposition.

3.1.1. Relative positions inside the towers. The definition of $G^{\epsilon, \delta}$ depends on whether $x \in Z_{l}^{(n-2)}$ or $x \in Z_{s}^{(n-2)}$. Throughout this section, the quantities $d(x)=d_{n-2}(x)$ and $h(x)=h_{n-2}(x)$ are defined as in Proposition 3.1 and locate the position of $x$ inside the tower of $\xi^{(n-2)}$ to which it belongs. If $x \in Z_{l}^{(n-2)}$, there exists $0 \leq j<q_{n-1}$ such that $x \in \Delta_{j}^{(n-2)}$; if $x_{0} \in \Delta_{0}^{(n-2)}$ is such that $R_{\alpha}^{j} x_{0}=x$, then $d_{n-2}(x)=x_{0}$ is the distance from 0 in the base floor. Similarly, if $x \in Z_{s}^{(n-2)}$, there exists $0 \leq j<q_{n-2}$ such that $x \in \Delta_{j}^{(n-1)}$ and if $z_{0} \in \Delta_{0}^{(n-1)}$ is such that $R_{\alpha}^{j} z_{0}=x$, then $d_{n-2}(x)=1-z_{0}$ is the distance in the base from 1 . The quantity $h_{n-2}(x)$, which is given respectively by $q_{n-1}-j$ or $q_{n-2}-j$, represents the distance of the floor to which $x$ belongs from the top of the tower. In particular, we remark that by construction $R_{\alpha}^{h_{n-2}(x)} x \in \Delta(n-2)$.

3.1.2. Cycles of order $n-2$. Let us use the notation $\mathcal{O}_{N}(x):=\left\{R_{\alpha}^{i} x, 0 \leq i<\right.$ $N\}$ to denote orbit segments. Let us first locate inside the orbit $\mathcal{O}_{N}(x)$ all orbit segments which correspond to cycles along towers of $\xi^{(n-2)}$. As just remarked,

\footnotetext{
${ }^{3}$ See 3.1 .1 below for a geometric explanation of the meaning of $d(x)$ and $h(x)$.
} 


$x_{0}^{(n-2)} x_{1}^{(n-2)} \quad x_{2}^{(n-2)} x_{\bar{c}_{n-2}-2}^{(n-2)} x_{\bar{c}_{n-2}-1}^{(n-2)} x_{\bar{c}_{n-2}}^{(n-2)}$

Figure 6. Decomposition of the orbit $\mathcal{O}_{N}(x)$ into cycles of order $n-2$.

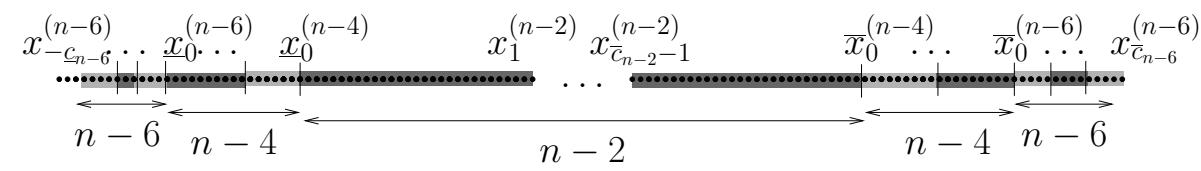

FiguRe 7. Decomposition of the orbit $\mathcal{O}_{N}(x)$ into cycles of further orders.

$R_{\alpha}^{h_{n-2}(x)} x \in \Delta(n-2)=\Delta^{(n-2)} \cup \Delta^{(n-1)}$ and $h_{n-2}(x)$ is the first time $i$ for which $R_{\alpha}^{i} x \in \Delta(n-2)$. Let $x_{0}^{(n-2)}:=R_{\alpha}^{h_{n-2}(x)} x$. The following visits to $\Delta(n-2)$ can be expressed though $T^{(n-2)}$, first return map to $\Delta(n-2)$ (see 1.1). Let

$x_{i}^{(n-2)}:=\left(T^{(n-2)}\right)^{i} x_{0}^{(n-2)} ; \quad \bar{c}_{n-2}:=\max \left\{i \in \mathbb{N}:\left(T^{(n-2)}\right)^{i} x_{0}^{(n-2)} \in \mathcal{O}_{N}(x)\right\}$, where $\bar{c}_{n-2}$ gives the number of visits of $\mathcal{O}_{N}(x)$ to $\Delta(n-2)$. Let $r_{n-2}(x)$ be the first return time of $x \in \Delta(n-2)$ to $\Delta(n-2)$, i.e. $r_{n-2}(x)=q_{n-1}$ if $x \in \Delta^{(n-2)}$, $r_{n-2}(x)=q_{n-2}$ if $x \in \Delta^{(n-1)}$. Then each orbit segment

$$
\mathcal{O}_{r_{n-2}\left(x_{i}^{(n-2)}\right)}\left(x_{i}^{(n-2)}\right)=\left\{R_{\alpha}^{i} x_{i}^{(n-2)}, 0 \leq i<r_{n-2}\left(x_{i}^{(n-2)}\right)\right\}
$$

is a cycle of order $n-2$ and all cycles corresponding to $i=0, \ldots, \bar{c}_{n-2}-1$ are completely contained in $\mathcal{O}_{N}(x)$, as in the representation of the orbit decomposition in Figure 6. Hence, so far

$$
\begin{aligned}
\mathcal{O}_{N}(x) & =\left\{R_{\alpha}^{i} x, 0 \leq i<h_{n-2}(x)\right\} \cup \bigcup_{i=0}^{\bar{c}_{n-2}-1} \mathcal{O}_{r_{n}\left(x_{i}^{(n-2)}\right)}\left(x_{i}^{(n-2)}\right) \\
& \cup\left\{R_{\alpha}^{i}(x), h_{n-2}(x)+\sum_{i=0}^{\bar{c}_{n-2}-1} r_{n-2}\left(x_{i}^{(n-2)}\right) \leq i<N\right\},
\end{aligned}
$$

where the orbit segments which appear in the central union are cycles of order $n-2$. We will refer to the first and the last term as to the initial and final orbit segments (see again Figure 6).

Let us estimate the number $\bar{c}_{n-2}$ of cycles of order $n-2$. Since the cardinality of points in a cycle of order $n-2$ is at least $q_{n-2}$ and $q_{n}>N$ definition of $n$, $\bar{c}_{n-2} \leq N / q_{n-2} \leq q_{n} / q_{n-2}$ and from the recurrent relation $q_{n}=a_{n} q_{n-1}+q_{n-2}$ we have the following.

Remark 3.2. $\bar{c}_{n-2} \leq\left(a_{n}+1\right)\left(a_{n-1}+1\right)$.

3.1.3. Further cycles. The initial and final segments of $\mathcal{O}_{N}(x)$ in (3.3) will be decomposed by induction into cycles of even orders $m<n-2$. Let $\bar{x}_{0}^{(n-4)}:=x_{\bar{c}_{n-2}}^{(n-2)}$ and $\underline{x}_{0}^{(n-4)}:=x_{0}^{(n-2)}$ be respectively the first point of the last segment and the last point of the initial segment. We remark that we have $\bar{x}_{0}^{(n-4)}, \underline{x}_{0}^{(n-4)} \in \Delta(n-4)$, since by construction of the partitions $\Delta(n-2) \subset \Delta(n-4)$. 
Assume by induction that we have already subdivided the initial and final segments into cycles up to order $m+2\left(m\right.$ even) and let $\bar{x}_{0}^{(m)}$ and $\underline{x}_{0}^{(m)}$ be respectively the last point of the initial segment and the first point of the final segment, with $\underline{x}_{0}^{(m)}, \bar{x}_{0}^{(m)} \in \Delta(m+2) \subset \Delta(m)$. Let

$$
\begin{array}{rlrl}
x_{i}^{(m)} & :=T^{(m)^{i}} \bar{x}_{0}^{(m)}, i=0, \ldots, \bar{c}_{m} ; & \bar{c}_{m}:=\max \left\{i \in \mathbb{N}: T^{(m)^{i}} \bar{x}_{0}^{(m)} \in \mathcal{O}_{N}(x)\right\} \\
x_{-i}^{(m)}:=T^{(m)}{ }^{-i} \underline{x}_{0}^{(m)}, i=1, \ldots, \underline{c}_{m} ; & \underline{c}_{m}:=\max \left\{i \in \mathbb{N}: T^{(m)^{-i}} \underline{x}_{0}^{(m)} \in \mathcal{O}_{N}(x)\right\} .
\end{array}
$$

The points $\left\{x_{i}^{(m)}, 0 \leq i \leq \bar{c}_{m}\right\}$ give all the visits to $\Delta(m)$ which occur in the final orbit segment and $\left\{x_{i}^{(m)},-\underline{c}_{m} \leq i \leq-1\right\}$ give all the visits to $\Delta(m)$ which occur in the initial orbit segment. Moreover, by construction,

$$
x_{i}^{(m)} \in \Delta(m) \backslash \Delta(m+2), \quad-\underline{c}_{m} \leq i \leq-1, \quad 1 \leq i \leq \bar{c}_{m} .
$$

Let $r_{m}(x)$ be as before the first return time of $x \in \Delta(m)$ to $\Delta(m)$. Thus, all orbit segments

$$
\mathcal{O}_{r_{m}\left(x_{i}^{(m)}\right)}\left(x_{i}^{(m)}\right), \quad i=0, \ldots, \bar{c}_{m}-1, \quad i=-\underline{c}_{m}, \ldots,-1
$$

are cycles of order $m$ which are completely contained in $\mathcal{O}_{N}(x)$. Moreover, since by (3.4) the initial and final segment do not contain any visit to $\Delta(m+2)$, except $\bar{x}_{0}^{(m)}$ and since the points in $[0,1) \backslash \Delta(m+2)$ have at least distance $\lambda^{(m+3)}$ from 0 and 1 , we also have the following.

Remark 3.3. The distance from 0 and from 1 of any of the points in the orbit segments $\mathcal{O}_{r_{m}\left(x_{i}^{(m)}\right)}\left(x_{i}^{(m)}\right)$ for $-\underline{c}_{m} \leq i \leq-1$ and $1 \leq i \leq \bar{c}_{m}-1$ is at least $\lambda^{(m+3)}$.

In the previous Remark, the orbit corresponding to $i=0$ was excluded since it contains $\bar{x}_{0}^{(m)} \in \Delta(m+2)$.

Let $\bar{x}_{0}^{(m-2)}:=x_{\bar{c}_{m}}^{(m)}$ and $\underline{x}_{0}^{(m-2)}:=x_{-\underline{c}_{m}}^{(m)}$ and continue by induction to decompose the remaining initial and final segments. If, for some $m$, we have $\underline{c}_{m}=\bar{c}_{m}=0$, there are no cycles of order $m$ in the decomposition. If either $\underline{c}_{m}$ or $\bar{c}_{m}$ are not zero, we say on the contrary that the order $m$ is present in the decomposition.

We get the following decomposition of the whole orbit into cycles:

$$
\mathcal{O}_{N}(x)=\bigcup_{\substack{m=0 \\ m \text { even }}}^{n-2} \bigcup_{i=-\underline{c}_{m}}^{\bar{c}_{m}-1} \mathcal{O}_{r_{m}\left(x_{i}^{(m)}\right)}\left(x_{i}^{(m)}\right),
$$

where for uniformity of notation, we set $\underline{c}_{n-2}:=0$ and the union for a given $m$ has to be considered empty when $\bar{c}_{m}=\underline{c}_{m}=0$. Since by construction the length of the initial and final segment after the decomposition of order $m+2$ is at most $q_{m+3}$ and each cycle of order $m$ has length at least $q_{m}$, using that $q_{m+1} / q_{m} \leq a_{m+1}+1$, we have the following.

Remark 3.4. The number $\bar{c}_{m}+\underline{c}_{m}$ of cycles of order $m$ in the decomposition satisfies

$$
\bar{c}_{m}+\underline{c}_{m} \leq 2\left(a_{m+3}+1\right)\left(a_{m+2}+1\right)\left(a_{m+1}+1\right)
$$


3.2. Reduction to finitely many cycles. The decomposition in 3.1 implies that:

$$
S_{N}(\alpha, x)=\sum_{\substack{m=0 \\ m \text { even }}}^{n(N, \alpha)-2} \sum_{i=-\underline{c}_{m}}^{\bar{c}_{m}-1} S_{r_{m}\left(x_{i}^{(m)}\right)}\left(\alpha, x_{i}^{(m)}\right) .
$$

In order to construct a good approximation of $\frac{1}{N} S_{N}\left(x_{0}, \alpha\right)$ in measure, it is enough to consider in the previous expression only a fixed and finite number of cycles.

Proposition 3.5. For each $\epsilon>0$ and $\delta>0$ there exist an even integer $M \geq 2$ and $N_{1} \in \mathbb{N}$ such that, for all $N \geq N_{1}$, we have

$$
\operatorname{Leb}\left\{(x, \alpha):\left|\frac{1}{N} S_{N}(\alpha, x)-\frac{1}{N} \sum_{\substack{m=n(N, \alpha)-M \\ m \text { even }}}^{n(N, \alpha)-2} \sum_{i=-\underline{c}_{m}}^{\bar{c}_{m}-1} S_{r_{m}\left(x_{i}^{(m)}\right)}\left(\alpha, x_{i}^{(m)}\right)\right| \geq \epsilon\right\} \leq \delta,
$$

where we adopt the convention that if $n(\alpha, N)<M$ the sum in (3.6) runs from $m=0$.

The reason why the Proposition holds is that the contributions from different cycles decay exponentially in the order. The set of small measure which needs to be neglected contains the set of $\alpha$ for which there are too many cycles of some orders and the set of initial points for which the contribution of the cycles of order $n-2$ is too large.

In the proof of Proposition 3.5 we will use the following.

Lemma 3.6. For each $\delta>0$ there exist a constant $C=C(\delta)$ and $N_{0}=N_{0}(\delta)>0$ so that for all $N \geq N_{0}$ there exists a set $A_{N}=A_{N}(\delta) \subset[0,1)$ such that $\operatorname{Leb}\left(A_{N}\right)<$ $\delta$ and, for all $\alpha \in[0,1) \backslash A_{N}$,

$$
a_{n(N, \alpha)-k} \leq C(k+1)^{2}, \quad 0 \leq k<n(N, \alpha) .
$$

The proof of Lemma 3.6 relies on the techniques used in [SU08. We postpone the proof to the Appendix, A.1.

Proof of Proposition 3.5. Let us consider the difference which we want to estimate to get (3.6). Let us denote, for brevity, $n=n(N, \alpha)$. From (3.5), applying Remark 3.4 to estimate the number of cycles of each order and keeping aside the term $i=0$, we get:

$$
\begin{gathered}
\left|\frac{1}{N} \sum_{\substack{m=0 \\
m \text { even }}}^{n-M-2} \sum_{i=-\underline{c}_{m}}^{\bar{c}_{m}-1} S_{r_{m}\left(x_{i}^{(m)}\right)}\left(\alpha, x_{i}^{(m)}\right)\right| \leq \frac{1}{N} \sum_{\substack{m=0 \\
m \text { even }}}^{n-M-2}\left|S_{r_{m}\left(x_{0}^{(m)}\right)}\left(\alpha, x_{0}^{(m)}\right)\right| \\
+\sum_{\substack{m=0 \\
m \text { even }}}^{n-M-2} \frac{r_{m}\left(x_{i}^{(m)}\right)}{N} 2 \prod_{s=1}^{3}\left(a_{m+s}+1\right) \frac{\left|S_{r_{m}\left(x_{i}^{(m)}\right)}\left(\alpha, x_{i}^{(m)}\right)\right|}{r_{m}\left(x_{i}^{(m)}\right)} .
\end{gathered}
$$

To estimate the contribution given by each cycle, let us apply Corollary 2.9, When $i \neq 0$, Remark 3.3 gives a lower bound on the contribution coming from closest points and we get

$$
\frac{\left|S_{r_{m}\left(x_{i}^{(m)}\right)}\left(\alpha, x_{i}^{(m)}\right)\right|}{r_{m}\left(x_{i}^{(m)}\right)} \leq \frac{1}{r_{m}\left(x_{i}^{(m)}\right) \lambda^{(m+3)}}+M \leq 2 \prod_{s=0}^{3}\left(a_{m+s}+1\right)+M, \quad i \neq 0,
$$


where in the last inequality we used that

$$
\frac{1}{r_{m}\left(x_{i}^{(m)}\right) \lambda^{(m+3)}} \leq \frac{1}{q_{m} \lambda^{(m-1)}} \prod_{s=0}^{3} \frac{\lambda_{m+s-1}}{\lambda^{m+s}} \leq 2 \prod_{s=0}^{3}\left(a_{m+s}+1\right) .
$$

Since $q_{n-2} \leq N, r_{m}\left(x_{i}^{(m)}\right) \leq q_{m+1}$ and $q_{n+2 s} \geq 2^{s} q_{n}$ (from the recurrent relations (1.3) $)$, assuming $M \geq 2$, we have

$$
\frac{r_{m}\left(x_{i}^{(m)}\right)}{N} \leq \frac{1}{2^{n-m-4}}, \quad 0 \leq m \leq n-4
$$

When $i=0$, for any order $m$ which is present in the decomposition (i.e. $\underline{c}_{m}>0$ ), let us estimate the contribution of the closest points to 0 and 1 in $\mathcal{O}_{r_{m}\left(x_{i}^{(m)}\right)}\left(x_{i}^{(m)}\right)$ with the distance from 0 and 1 of elements of the last (i.e. the minimum $m^{\prime}>m$ ) order $m^{\prime}$ which is present. To get an upper estimate, let us consider the worst case in which all orders $m<n-M$ are present. For $m<n-M-2$, a lower bound for minimum distance from 0 or 1 of points of order $m+2$ (which belong to $[0,1) \backslash \Delta(m+4)$ by (3.4) $)$ is given by $\lambda^{(m+5)}$. For $m=n-M-2$, let us denote the minimum between the distance of $x_{0}^{(n-M-2)}$ from 0 and 1 by $m_{n-M-2}(x)$. Thus, applying Corollary 2.9 we get

$$
\begin{aligned}
& \left|\sum_{\substack{m=0 \\
m \text { even }}}^{n-M-2} \frac{S_{r_{m}\left(x_{0}^{(m)}\right)}\left(\alpha, x_{0}^{(m)}\right)}{N}\right| \leq \\
& \frac{1}{N m_{n-M+2}(x)}+\sum_{\substack{m=0 \\
m \text { even }}}^{n-M-4} \frac{r_{m}\left(x_{i}^{(m)}\right)}{N} \frac{1}{r_{m}\left(x_{i}^{(m)}\right) \lambda^{(m+5)}}+\sum_{\substack{m=0 \\
m \text { even }}}^{n-M-2} \frac{r_{m}\left(x_{i}^{(m)}\right)}{N} M \leq \\
& \frac{1}{N m_{n-M+2}(x)}+\sum_{\substack{m=0 \\
m \text { even }}}^{n-M-4} \frac{2 \prod_{s=0}^{5}\left(a_{m+s}+1\right)}{2^{n-m-4}}+\sum_{\substack{m=0 \\
m \text { even }}}^{n-M-2} \frac{M}{2^{n-m-4}}
\end{aligned}
$$

where in the last inequality we used (3.11) and an estimate analogous to (3.10).

Combining (3.9), (3.11) and (3.12) we have the following upper estimate for (3.8):

$$
\begin{aligned}
& \sum_{\substack{m=0 \\
m \text { even }}}^{n-M-2} \frac{2 \prod_{s=1}^{3}\left(a_{m+s}+1\right)\left(M+2 \prod_{s=0}^{3}\left(a_{m+s}+1\right)\right)+M}{2^{n-m-4}}+ \\
& \quad+\sum_{\substack{m=0 \\
m \text { even }}}^{n-M-4} \frac{2 \prod_{s=0}^{5}\left(a_{m+s}+1\right)}{2^{n-m-4}}+\frac{1}{N m_{n-M+2}(x)} .
\end{aligned}
$$

Let us now prove (3.6). Fix $\epsilon>0, \delta>0$. By Lemma 3.6, for some $N_{0}$, we can choose for each $N \geq N_{0}$, a set $A_{N} \subset[0,1)$ such that $\operatorname{Leb}\left(A_{N}\right)<\delta / 2$ and if $\alpha \notin A_{N}, a_{n-k} \leq C(k+1)^{2}$, for $n=n(N, \alpha)$. Let us estimate (3.13). Since all terms are positive, we get an upper estimate by making both series run from 0 to $n-M-2$. Hence, the first term in (3.13), defining for brevity $L\left(x_{0}, x_{1}, \ldots, x_{5}\right)=$ $2 \prod_{s=1}^{3}\left(x_{s}+1\right)\left(M+2 \prod_{s=0}^{3}\left(x_{s}+1\right)\right)+M+2 \prod_{s=0}^{5}\left(x_{s}+1\right)$, can be written and 
estimated as follows:

$$
\begin{aligned}
\sum_{\substack{m=0 \\
m \text { even }}}^{n-M-2} \frac{L\left(a_{m}, \ldots, a_{m+5}\right)}{2^{n-m-4}} & \leq \sum_{\substack{m=0 \\
m \text { even } \\
n-M-2}}^{n-M-2} \frac{L\left(C(n-m+1)^{2}, \ldots, C(n-m-4)^{2}\right)}{2^{n-m-4}} \leq \\
& \leq \sum_{\substack{m=0 \\
m \text { even }}}^{n-P(n-m) \mid} \leq \sum_{\substack{k=M+2 \\
k \text { even }}}^{\infty} \frac{|P(k)|}{2^{k-4}}
\end{aligned}
$$

for some polynomial $P(x)$. Hence, choosing $M$ large enough, we can assure that the remainder of the series is less than $\epsilon / 2$.

In order to conclude the proof, we still need to estimate the second term in (3.13). Let us first estimate the expectation of the quantity $\left(\mathrm{Nm}_{n-M+2}(x)\right)^{-1}$. Let $E$ denote the conditional expectation with respect to the Lebesgue measure on the $x$ variable, for $\alpha$ and $N$ (and hence $n=n(N, \alpha)$ ) fixed. Let us show that there exists a set $X_{n} \subset[0,1)$, with $\operatorname{Leb}\left(X_{n}\right) \leq \delta / 4$, such that, if $\chi_{X_{n}^{C}}$ denotes the characteristic function of $X_{n}^{C}$,

$$
E\left(\frac{\chi_{X_{n}^{C}}(x)}{N m_{n-M+2}(x)}\right) \stackrel{M \rightarrow \infty}{\longrightarrow} 0 .
$$

Let us recall that $m_{n-M-2}(x)=\min \left\{x_{0}^{(n-M-2)}, 1-x_{0}^{(n-M-2)}\right\}$ and that $x_{0}^{(n-M-2)}=$ $x_{\bar{c}_{n-M}}^{(n-M)}$. Since by construction $x_{\bar{c}_{n-M}}^{(n-M)}$ is the last visit to $\Delta(n-M)$ in $\mathcal{O}_{x}(N)$ and such visits are not more than $q_{n-M+1}$ apart, we have $x_{0}^{(n-M-2)} \in\left\{R_{\alpha}^{N-i} x: 1 \leq\right.$ $\left.i \leq q_{n-M+1}\right\}$ and hence

$$
m_{n-M-2}(x) \geq \min \left\{R_{\alpha}^{N-i} x, 1-R_{\alpha}^{N-i} x, 1 \leq i \leq q_{n-M+1}\right\} .
$$

Consider the set

$$
X_{n}=\bigcup_{i=1}^{q_{n}-M+1} R_{\alpha}^{-N+i}\left(\left[0, \frac{\delta}{8 q_{n-M+1}}\right] \cup\left[1-\frac{\delta}{8 q_{n-M+1}}, 1\right]\right) .
$$

Clearly $\operatorname{Leb}\left(X_{n}\right) \leq \delta / 4$ and if $x \notin X_{n}$, by (3.15) we have $m_{n-M-2}(x) \geq \delta / 8 q_{n-M+1}$. Hence, since $q_{n-2}<N$,

$$
E\left(\frac{\chi_{X_{n}^{C}}(x)}{N m_{n-M+2}(x)}\right) \leq E\left(\frac{\chi_{X_{n}^{C}}(x)}{q_{n-2} m_{n-M+2}(x)}\right) \leq \frac{8}{\delta} \frac{q_{n-M+1}}{q_{n-2}},
$$

from which (3.14) follows using that $q_{n+2 s} \geq 2^{s} q_{n}$ by the recursive relations (1.3).

Hence, enlarging again $M$ if necessary, we can hence assure that the expectation in (3.14) is less than $\epsilon \delta / 8$. Remark moreover that the choice of $M$ depends on $\epsilon$ and $\delta$ only and is uniform in $n$ and $N$.

Let us denote by $X_{n}^{\prime}=\left\{x: \frac{1}{N m_{n-M+2}(x)} \geq \epsilon / 2\right\}$. Thus, using Chebyshev inequality, we get that

$$
\operatorname{Leb}\left(X_{n}^{\prime}\right) \leq \frac{E\left(\frac{\chi_{X_{n}^{C}}(x)}{N m_{n-M+2}(x)}\right)}{\epsilon / 2}+\operatorname{Leb}\left(X_{n}\right) \leq \frac{\delta}{2} .
$$

This shows that also the second term of (3.13) is $\epsilon / 2$-small when $\alpha \notin A_{N}$ and $x \notin X_{n(\alpha, N)}^{\prime}$ and hence concludes the proof of the Lemma. 
Proof of Proposition 3.1. Let us define the function $G^{\epsilon, \delta}$ using the truncated decomposition into cycles. Given $\epsilon$ and $\delta$, let $M$ be given by Proposition 3.5 applied to $\epsilon / 2$ and $\delta / 2$ and let $g_{n}^{\varepsilon}$ and $K$ be as in Proposition 2.1, relative to some $\varepsilon$ which will be fixed below as a function of $\epsilon$. Then define

$$
G^{\epsilon, \delta}(x, \alpha, N):=\sum_{\substack{m=n(\alpha, N)-M \\ m \text { even }}}^{n(\alpha, N)-2} \sum_{i=-\underline{c}_{m}}^{\bar{c}_{m}-1} \frac{r_{m}\left(x_{i}^{(m)}\right)}{N} g_{m}^{\varepsilon}\left(\alpha, x_{i}^{(m)}, r_{m}\left(x_{i}^{(m)}\right)\right)
$$

with the convention that the sum runs from $m=0$ if $n(\alpha, N)<M$. Let $K_{1}=$ $M+K$. The estimate on $r_{m}\left(x_{i}^{(m)}\right) / N$ given by (3.11) holds for $m \leq n-4$. Hence, by Proposition 2.1 and Remarks 3.2 and 3.4 on the complement, denoting $n=n(N, \alpha)$, we have

$$
\begin{aligned}
& \left|G^{\epsilon, \delta}(x, \alpha, N)-\sum_{\substack{m=n-M \\
m \text { even }}}^{n-2} \sum_{i=-\underline{c}_{m}}^{\bar{c}_{m}-1} \frac{r_{m}\left(x_{i}^{(m)}\right)}{N} \frac{S_{r_{m}\left(x_{i}^{(m)}\right)}\left(\alpha, x_{i}^{(m)}\right)}{r_{m}\left(x_{i}^{(m)}\right)}\right| \leq \\
& \leq \varepsilon\left(\frac{q_{n-1}}{N}\left(a_{n}+1\right)\left(a_{n-1}+1\right)+\sum_{\substack{m=n-M \\
m \text { even }}}^{n-4} \frac{2 \prod_{s=0}^{3}\left(a_{m+s}+1\right)}{2^{n-m-4}}\right) .
\end{aligned}
$$

By Theorem 3, neglecting a subset of $\alpha$ of Lebesgue measure less than $\delta / 2$, we can also assume that $q_{n} / N \leq C$ and $a_{n}, a_{n-1} \leq A$ for some $C>1, A>1$, so that the first term in the upper bound of (3.17) is less than $C(A+1)^{2}$. If moreover $\alpha \notin A_{N}$ where $A_{N}=A_{N}(\delta / 2)$ is the set given by Lemma 3.6, since, reasoning again as in the proof of Proposition 3.5, the terms at numerators in the RHS are bounded by a polynomial in $(n-m)$, the series in the RHS is converging. Thus, choosing $\varepsilon$ appropriately, (3.17) is less than $\epsilon / 2$. Combining (3.17) with Proposition 3.5, we proved (3.1).

Let us show that $G^{\epsilon, \delta}$ can be expressed as a function of the variables in (3.2). The variable $q_{n} / N$ already appears explicitly in front of the sum. Let us show that, for each $m$ involved in the sum, the quantities $\underline{c}_{m}, \bar{c}_{m}$ and $x_{i}^{(m)}, r_{m}\left(x_{i}^{(m)}\right)$ for $-\underline{c}_{m} \leq i \leq \bar{c}_{m}$, as they appear in the sum, can be expressed in the desired form.

Let us use induction on $m$. When $m=n-2:=n(\alpha, N)-2$, if we denote $x_{-1}^{(n-2)}:=$ $\left(T^{(n-2)}\right)^{-1} x_{0}^{(n-2)}$, we have by construction that $x_{-1}^{(n-2)}=d(x)$ or $1-d(x)$, according to whether $x \in Z_{l}^{(n-2)}$ or $Z_{s}^{(n-2)}$. The other points $x_{i}^{(n-2)}, i \geq 0$, are completely determined by the orbit under $T^{(n-2)}$. Hence, the ratios $x_{i}^{(n-2)} / \lambda^{(n-2)}$ (which appear as variables of the functions $g_{n-2}^{\epsilon}$, see Proposition 2.11) are determined by $d(x) / \lambda^{(n-2)}$ (or $d(x) / \lambda^{(n-1)}$, according to whether $x \in Z_{l}^{(n-2)}$ or $Z_{s}^{(n-2)}$ ) and $\lambda^{(n-1)} / \lambda^{(n-2)}$. The other variables of $g_{n-2}^{\epsilon}$, i.e. $\left(\lambda^{(n-2)} q_{n-1}\right)^{-1}\left(\right.$ or $\left.\left(\lambda^{(n-1)} q_{n-2}\right)^{-1}\right)$ and $a_{n+2-s}, 0 \leq s \leq K$, are already given, since $K \leq K_{1}$. Similarly, the orbit under the map $T^{(n-2)}$ determines also whether $x_{i}^{(n-2)} \in \Delta_{0}^{(n-1)}$ or $x_{i}^{(n-2)} \in \Delta_{0}^{(n-2)}$ and therefore $r_{n-2}\left(x_{i}^{(n-2)}\right)$. The number of iterations $\bar{c}_{n-2}$ can be expressed as the maximum $c$ such that $h(x)+\sum_{i=1}^{c} r_{n-2}\left(x_{i}^{(n-2)}\right) \leq N$, hence it involves ratios of type $q_{n-2} / N$ and $q_{n-1} / N$ (or equivalently $q_{n} / N$ and $q_{n-2} / q_{n-1}$ and $a_{n}$ ) and $h(x) / N$. This concludes the base of the induction. 
For the inductive step, from $m+2$ to $m$, we remark, as before, that $x_{i}^{(m)}$ and $r_{m}\left(x_{i}^{(m)}\right)$ are completely determined by the initial points $\bar{x}_{0}^{(m)}$ and $\underline{x}_{0}^{(m)}$ and by the positive and negative orbit of the induced map $T^{(m)}$. Moreover, the ratio $\lambda^{(m+1)} / \lambda^{(m)}$ is determined by the previous ratio $\lambda^{(m+3)} / \lambda^{(m+2)}$ and the entries $a_{m+2}, a_{m+3}$, while $\bar{x}_{0}^{(m)} / \lambda^{(m)}$ and $\underline{x}_{0}^{(m)} / \lambda^{(m)}$ (or respectively $\bar{x}_{0}^{(m)} / \lambda^{(m+1)}$ and $\left.\underline{x}_{0}^{(m)} / \lambda^{(m+1)}\right)$ can be obtained from $x_{\bar{c}_{m+2}}^{(m+2)} / \lambda^{(m+2)}$ and $x_{-\underline{c}_{m+2}}^{(m+2)} / \lambda^{(m+2)}$ (or $x_{\bar{c}_{m+2}}^{(m+2)} / \lambda^{(m+3)}$ and $\left.x_{-\underline{c}_{m+2}}^{(m+2)} / \lambda^{(m+3)}\right)$, given by the inductive step, and $\lambda^{(m+2)} / \lambda^{(m)}$. In particular, the function $g_{m}^{\varepsilon}$ is a function ratios which can be expressed in terms of the above quantities. Reasoning as for $m=n-2$, also $r_{m}\left(x_{i}^{(m)}\right)$ are determined by the orbit of the induced map $T^{(m)}$. Analyzing the decomposition in $\$ 3.1$, one can see that the numbers $\underline{c}_{m}$ and $\bar{c}_{m}$ of cycles can be expressed respectively as the biggest integers $\underline{c}$ and $\bar{c}$ such that

$$
\begin{gathered}
\sum_{\substack{m+2 \leq k \leq n-4 \\
k \text { even }}} \sum_{i=-\underline{c}_{k}}^{-1} r_{k}\left(x_{i}^{(k)}\right)+\sum_{i=-\underline{c}}^{-1} r_{m}\left(x_{i}^{(m)}\right) \leq h(x) \\
\sum_{\substack{m+2 \leq k \leq n-2 \\
k \text { even }}} \sum_{i=0}^{\bar{c}_{k}-1} r_{k}\left(x_{i}^{(k)}\right)+\sum_{i=0}^{\bar{c}-1} r_{m}\left(x_{i}^{(m)}\right)<N-h(x) .
\end{gathered}
$$

Hence, dividing by $N$, one sees that they are determined by ratios which, by inductive assumption, are already expressed as desired and by ratios of type $q_{m} / N$ and $q_{m+1} / N$, which can be determined from them, involving also $a_{m+2}, a_{m+3}$. The other variables which appear in $g_{m}^{\varepsilon}$ by Proposition 2.1 are $a_{m+2-s}$ for $0 \leq s \leq K$, $n-M \leq m<n$, which are included as variables thanks to the definition of $K_{1}$. This concludes the induction.

\section{Existence of the limiting distribution.}

Limiting distributions of relative positions in the towers. Let us consider the variables from which $G^{\epsilon, \delta}$ depends (see (3.2)). Give $\alpha, N$, let $n=n(N, \alpha)$ and let $d(x)=d_{n-2}(x)$ and $h(x)=h_{n-2}(x)$ be defined as in 3 . Consider the random variables on $[0,1) \times[0,1)$

$$
\begin{aligned}
& D_{N}(x, \alpha)=\frac{d_{n(N, \alpha)-2}(x)}{\lambda^{(n-2)}} \chi_{Z_{l}^{(n-2)}}(x, \alpha)+\frac{d_{n(N, \alpha)-2}(x)}{\lambda^{(n-1)}} \chi_{Z_{s}^{(n-2)}}(x, \alpha) \\
& H_{N}(x, \alpha)=\frac{h_{n(N, \alpha)-2}(x)}{q_{n-1}} \chi_{Z_{l}^{(n-2)}}(x, \alpha)+\frac{h_{n(N, \alpha)-2}(x)}{q_{n-2}} \chi_{Z_{s}^{(n-2)}}(x, \alpha) \\
& T_{N}(x, \alpha)=\left(q_{n-1} \lambda^{(n-2)}\right)^{-1} \chi_{Z_{l}^{(n-2)}}(x, \alpha)+\left(q_{n-2} \lambda^{(n-1)}\right)^{-1} \chi_{Z_{s}^{(n-2)}}(x, \alpha)
\end{aligned}
$$

where $\chi_{Z_{\omega}^{(n-2)}}(\omega=l$ or $s)$ denotes the indicator function of the towers, i.e.

$$
\chi_{Z_{\omega}^{(n-2)}}(x, \alpha)= \begin{cases}1 & \text { iff } x \in Z_{\omega}^{(n-2)}, \quad n=n(N, \alpha) ; \\ 0 & \text { otherwise. }\end{cases}
$$

The quantities $D_{N}$ and $H_{N}$ locate the relative position of $x$ inside the tower of $\xi^{(n-2)}$ to which it belongs, while $T_{N}$ give the total measure of the tower. We also remark that $D_{N}, H_{N}, T_{N}$ can be used as variables in the expression for $G^{\epsilon, \delta}$ given in 3.2 . 
Lemma 4.1. $D_{N}(x, \alpha), H_{N}(x, \alpha)$ and $T_{N}(x, \alpha)$ have limiting distributions as $N$ tend to infinity.

Proof. Since $x$ is uniformly distributed, on each event $\left\{(x, \alpha) \in Z_{w}^{(n-2)}\right\}$, $w=l, s$, the random variables $d_{n(N, \alpha)}(x)$ and $h_{n(N, \alpha)}(x)$ are renormalized so that $D_{N}(x, \alpha)$ and $H_{N}(x, \alpha)$ are uniformly distributed on $[0,1)$ for each $N$. Since $\operatorname{Leb}\left\{x \in Z_{l}^{(n-2)}\right\}=\lambda^{(n-2)} q_{n-1}$ and $\operatorname{Leb}\left\{x \in Z_{s}^{(n-2)}\right\}=\lambda^{(n-1)} q_{n-2}$, the existence of the limiting distribution of $T_{N}$ follows from Corollary 1.3 .

\subsection{Tightness and final arguments.}

Lemma 4.2. The random variables $G^{\epsilon, \delta}$ are uniformly tight in $\epsilon, \delta$ and $N$, i.e.

$$
\inf _{\substack{\epsilon, \delta<1 \\ N \in \mathbb{N}}} \operatorname{Leb}\left\{(x, \alpha): G_{N}^{\epsilon, \delta}(\alpha, x, N) \leq T\right\} \stackrel{T \rightarrow+\infty}{\longrightarrow} 1 .
$$

Proof. Let us remark that, from the definition of $G^{\epsilon, \delta}$ in (3.16), as $\epsilon$ and $\delta$ tends to zero (and hence $M \rightarrow \infty$ ), $G^{\epsilon, \delta}$ has the same structure, but more and more terms are present in the series while at the same time each function $g_{m}^{\varepsilon}$ in the series involves more and more variables (i.e. $K \rightarrow \infty$ in (2.1)).

Since $\inf _{\epsilon, \delta, N} \operatorname{Leb}\left\{G_{N}^{\epsilon, \delta} \leq T\right\} \geq \operatorname{Leb}\left\{\sup _{\epsilon, \delta, N} G_{N}^{\epsilon, \delta} \leq T\right\}$, it is enough to estimate the latter. Let us estimate $G^{\epsilon, \delta}$ arguing as in the proof of Proposition 3.5 to prove (3.13), hence using (3.11), Remark 3.4 and moreover (2.2) in order to apply Corollary 2.9. For each $\delta>0$, we can find by Lemma 3.6 a $C(\delta)>1$ and set $A_{N}(\delta) \subset[0,1)$ on which (3.7) holds for $N$ sufficiently large and, by Theorem 3 a $C^{\prime}=C^{\prime}(\delta)>0$ and $A_{N}^{\prime}(\delta) \subset[0,1)$ such that $q_{n} / N \leq C^{\prime}$ and $a_{n}, a_{n-1} \leq C^{\prime}$ on $[0,1) \backslash A_{N}^{\prime}$.

Hence, for each $\alpha \notin A_{N} \cup A_{N}^{\prime}$,

$$
\begin{aligned}
& \sup _{\substack{\epsilon<1 \\
\delta<1}}\left|G^{\epsilon, \delta}(x, \alpha, N)\right| \leq \sum_{\substack{m=0 \\
m \text { even }}}^{n-2} \sum_{i=-\underline{c}_{m}}^{\bar{c}_{m}-1} \frac{r_{m}\left(x_{i}^{(m)}\right)}{N} \sup _{\varepsilon<1}\left|g_{m}^{\varepsilon}\left(\alpha, x_{i}^{(m)}, r_{m}\left(x_{i}^{(m)}\right)\right)\right| \\
& \leq \sum_{\substack{m=0 \\
m \text { even }}}^{n-2} \sum_{i=-\underline{c}_{m}}^{\bar{c}_{m}-1} \frac{r_{m}\left(x_{i}^{(m)}\right)}{N} \sup _{\varepsilon<1}\left(\varepsilon+\left|\frac{S_{r_{m}\left(x_{i}^{(m)}\right)}\left(\alpha, x_{i}^{(m)}\right)}{r_{m}\left(x_{i}^{(m)}\right)}\right|\right) \\
& \leq \sum_{\substack{m=0 \\
m \text { even }}}^{n-4} \frac{\left|P\left(C(n-m)^{2}\right)\right|}{2^{n-m-4}}+C^{\prime}\left(C^{\prime}+1\right)^{2}\left(1+M+\frac{1}{q_{n-1} m_{n-2}(x)}\right),
\end{aligned}
$$

where $P(x)$ is a fixed polynomial and $m_{n-2}(x)=\min \left\{T^{i} x, 1-T^{i} x ; 0 \leq i<N\right\}$. Let $X_{\nu}=\left\{x: m_{n-2}(x) q_{n-1} \leq \nu\right\}$. Hence, if $\alpha \notin A_{N} \cup A_{N}^{\prime}$ and $x \notin X_{\nu}$, since the series in (4.5) is converging, $G_{\epsilon, \delta}(\alpha, x, N)$ is uniformly bounded by a constant $T=T\left(C, C^{\prime}, 1 / \nu\right)$ which depends on $C, C^{\prime}, 1 / \nu$. Since $X_{\nu}$ is such that $\operatorname{Leb}\left(X_{\nu}\right) \rightarrow 0$ as $\nu \rightarrow 0$ uniformly in $n$ (for $\alpha \notin A_{N^{\prime}}$ ) and since moreover, from Lemma 3.6 and from Theorem $3 \operatorname{Leb}\left(A_{N}(\delta)\right) \rightarrow 0$ and $\operatorname{Leb}\left(A_{N}^{\prime}(\delta)\right) \rightarrow 0$ as $\delta \rightarrow 0$, which correspond to choosing $C^{\prime}(\delta)$ and $C(\delta)$ sufficiently large, this is enough to conclude the proof.

Recall that fixed $\epsilon>0, \delta>0, G^{\epsilon, \delta}$ can be expressed as a function of finitely many random variables, listed in (3.2). Some of them can be expressed through 
the random variables in (4.1, 4.2, 4.3) and all of them have a limiting distribution, either by Corollary 1.3 or by Lemma 4.1 .

Lemma 4.3. For any $\epsilon>0$ and $\delta>0$, let $D$ be the set such that $G^{\epsilon, \delta}$ is discontinuous as a function of the random variables in (3.2). Then the set of $(x, \alpha)$ such that the limits of the random variables in (3.2) belong to $D$ has measure zero.

Proof. Among the random variables in (3.2), expressed through 4.1, 4.2 4.3), only two depend on $x$, i.e. $D_{N}$ and $H_{N}$. For each given value of all the other ones, one can see that there are only finitely many values of these two, near which $G^{\epsilon, \delta}$ changes discontinuously: more precisely, discontinuities might happen only when $d(x)$ and $h(x)$ correspond to discontinuities $x$ of the induced maps $T^{m}$ for $n-M \leq m \leq n-2$. Hence, the set $D$ has measure zero in the domain of $G^{\epsilon, \delta}$. Since $D_{N}$ and $H_{N}$ are uniform random variables and have a uniformly distributed limit, also the set of $(x, \alpha)$ which are mapped to $D$ by their limit has measure zero.

Proof of Theorem 2, Let $f=f_{1}+f_{2}$ satisfy the assumptions of the Theorem. Since $f_{2}$ is integrable and for all $\alpha \in[0,1) \backslash \mathbb{Q}$ the rotation $R_{\alpha}$ is ergodic, by Birkhoff ergodic theorem, for a.e. $(x, \alpha)$ the Birkhoff sums $S_{N}\left(\alpha, x, f_{2}\right) / N$ converge to the constant $\int f_{2}$. In particular, a.e. convergence implies convergence in distribution. Let us hence consider separately the Birkhoff sums $S_{N}\left(\alpha, x, f_{1}\right)$ of $f_{1}$.

To show that $S_{N}\left(x, \alpha, f_{1}\right) / N$ has a limiting distribution, it is enough to show that for each continuous and bounded function $g$, if $E$ denotes the expectation with respect to the Lebesgue measure on $(x, \alpha), \lim _{N \rightarrow \infty} E g\left(S_{N}\left(x, \alpha, f_{1}\right) / N\right)=E g(S)$ for some random variable $S$.

Let us first show that, for each $\epsilon>0, \delta>0, G^{\epsilon, \delta}(\cdot, \cdot, N)$ has a limiting distribution as $N$ tends to infinity. By Proposition 3.1 . $G^{\epsilon, \delta}$ can be expressed as a function of variables which, by Corollary 1.3 and by Lemma 4.1 have all a limiting distribution as $N$ tends to infinity. The condition on the discontinuity sets proved in Lemma 4.3 is exactly what guarantees, by a standard result (see e.g. Theorem 2.1, Chapter III $\S 8$ in Shi96]), that also $G^{\epsilon, \delta}$ has a limiting distribution.

Hence, for some random variable $S^{\epsilon, \delta}, \lim _{N \rightarrow \infty} E g\left(G^{\epsilon, \delta}(\cdot, \cdot, N)\right)=E g\left(S^{\epsilon, \delta}\right)$ for each choice of $\epsilon$ and $\delta$, where $g$ is as before any bounded and continuous function. Using the tightness in Lemma 4.2 and Prokhorov's theorem, one can show that there exists a subsequence $S^{\epsilon_{k}, \delta_{k}}$ which converge in distribution to some $S$.

Let us prove the convergence in distribution of $S_{N} / N$. Given $g$ bounded and continuous and $\varepsilon>0$, by the previous paragraph we can choose $k_{0}$ sufficiently large so that $\left|E g\left(S^{\epsilon_{k_{0}}}, \delta_{k_{0}}\right)-E g(S)\right| \leq \varepsilon / 3$. We can estimate

$$
\begin{aligned}
& \left|E g\left(\frac{S_{N}\left(\cdot, \cdot, f_{1}\right)}{N}\right)-E g(S)\right| \leq E\left|g\left(\frac{S_{N}\left(\cdot, \cdot, f_{1}\right)}{N}\right)-g\left(G^{\epsilon_{k_{0}}, \delta_{k_{0}}}(\cdot, \cdot, N)\right)\right|+ \\
& +\left|E g\left(G^{\epsilon_{k_{0}}, \delta_{k_{0}}}(\cdot, \cdot, N)\right)-E g\left(S^{\epsilon_{k_{0}}, \delta_{k_{0}}}\right)\right|+\left|E g\left(S^{\epsilon_{k_{0}}, \delta_{k_{0}}}\right)-E g(S)\right| .
\end{aligned}
$$

By choice of $k_{0}$, the last term in (4.7) is less than $\varepsilon / 3$. By the previous arguments, there exists some $N_{1}>0$, such that for all $N \geq N_{1}$ also the second term in (4.7) is less than $\varepsilon / 3$. Substituting $k_{0}$ with a bigger one if necessary, we can assume that $\delta_{k_{0}} \leq \varepsilon / 12 \max g$. Moreover, if $k_{0}$ is large enough, using absolute continuity of $g$ on a compact set given by tightness, by Proposition 3.1 there exists $N_{2}$ such that if $N \geq N_{2}$, the RHS term in (4.6) is controlled by $\delta_{k_{0}} 2 \max g+\varepsilon / 6$. Hence for each $N \geq \max \left(N_{1}, N_{2}\right)$, the LHS of (4.6) is less than $\varepsilon$. This concludes the proof. 


\section{Appendix A.}

A.1. Proof of Lemma 3.6. We present here the proof of Lemma 3.6, which is based on the techniques and results used in [SU08. We just briefly recall the notation referring to the paper [SU08 for further details.

As in SU08, let $\alpha \mapsto \mathscr{G}(\alpha)=\left\{\frac{1}{\alpha}\right\}$ be the Gauss map and $\mu_{1}$ its invariant measure given by the density $\frac{\mathrm{d} \mu_{1}}{\mathrm{~d} \alpha}=(\ln 2(1+\alpha))^{-1}$. Let $\hat{\mathscr{G}}$ be the natural extension of $\mathscr{G}$, which acts as a shift on bi-infinite sequences $\hat{\alpha}=\left\{a_{n}\right\}_{n \in \mathbb{Z}}=\left(\hat{\alpha}^{-}, \hat{\alpha}^{+}\right)$and let $\mu_{2}$ be the natural invariant measure for $\hat{\mathscr{G}}$, which satisfies $\mu_{2}=\pi_{*} \mu_{1}$ where $\pi: \hat{\alpha}=\left\{a_{n}\right\}_{n \in \mathbb{Z}} \mapsto \alpha=\hat{\alpha}^{+}=\left\{a_{n}\right\}_{n \in \mathbb{N}}$ is the natural projection. Let $\left\{\Phi_{t}\right\}_{t \in \mathbb{R}}$ denote the special flow built over $\hat{\mathscr{G}}$ under the roof function $\varphi(\hat{\alpha})=-\ln \left(\hat{\mathscr{G}}(\hat{\alpha})^{-}\right)$ and let $\mu_{3}$ denote the measure given by $\mathrm{d} \mu_{3}=\mathrm{d} \mu_{2} \mathrm{~d} z$ on the domain $D$ of $\left\{\Phi_{t}\right\}_{t \in \mathbb{R}}$. As shown in $\S 4$ in [SU08, $\left\{\Phi_{t}\right\}_{t \in \mathbb{R}}$ is mixing.

Proof of Lemma 3.6. Let $\hat{A}_{k}=\left\{\hat{\alpha}: a_{-k} \leq(k+1)^{2}\right\}$. It is easy to check that $\left.\sum_{k=0}^{\infty} \mu_{2}\left(\hat{A}_{k}^{C}\right)=\sum_{k=0}^{\infty} \mu_{1}\left\{\alpha: a_{1} \geq(k+1)^{2}\right\}\right)<\infty$. Hence, by Borel-Cantelli Lemma, for a.e. $\hat{\alpha}$ there exists $c=c(\hat{\alpha})>0$ such that $a_{-k} \leq c(k+1)^{2}$ for all $k \in \mathbb{N}$. Moreover, given $\delta^{\prime}$, we can find $\hat{A}=\hat{A}\left(\delta^{\prime}\right)$ and $C=C\left(\delta^{\prime}\right)$ such that uniformly, for each $\hat{\alpha} \notin \hat{A}$ and $k \in \mathbb{N}, a_{-k} \leq C(k+1)^{2}$.

Since the condition (3.7) depends only on $a_{n}$ with $n>0$, i.e. it is invariant on fibers $\pi^{-1}(\alpha)$, for any $c>0$, setting $n(N, \hat{\alpha})=n(N, \pi \hat{\alpha})$, we have

$$
\begin{aligned}
\mu_{1}(\{\alpha: & \left.a_{n(N, \alpha)-k} \leq c(k+1)^{2}, \quad 0 \leq k<n(N, \alpha)\right\}= \\
= & \mu_{2}\left(\left\{\hat{\alpha}: a_{n(N, \hat{\alpha})-k} \leq c(k+1)^{2}, \quad 0 \leq k<n(N, \alpha)\right\} \geq\right. \\
\geq & \mu_{2}\left(\left\{\hat{\alpha}: a_{n(N, \hat{\alpha})-k} \leq c(k+1)^{2}, \quad k<n(N, \alpha), k \in \mathbb{Z}\right\},\right.
\end{aligned}
$$

where the last inequality follows from the inclusions between the two sets. In order to conclude the proof, we want to show that for some $c>0$, A.1 is bigger than $1-\delta$ for all $N$ sufficiently large. To prove it, we will use mixing of the special flow $\left\{\Phi_{t}\right\}_{t \in \mathbb{R}}$.

The set in A.1) contains $\left\{\hat{\alpha}: \hat{\mathscr{G}}^{n(N, \hat{\alpha})}(\hat{\alpha}) \notin \hat{A}\left(\delta^{\prime}\right)\right\}$ if we take $c=C\left(\delta^{\prime}\right)$. Let us localize the set of $\hat{\alpha}$ considered so that we describe the set through the flow $\left\{\Phi_{t}\right\}_{t \in \mathbb{R}}$. Reasoning as in the proof of Theorem 1.1 in $\mathbf{S U 0 8}$, for each $\varepsilon>0$ we can construct a finite union of cylinders $\mathcal{C}$ and subsets $U_{\mathcal{C}} \subset \mathcal{C}$ such that $\sum_{\mathcal{C}} \mu_{2}\left(U_{\mathcal{C}}\right) \geq 1-\varepsilon$ and for all $\hat{\alpha} \in U_{\mathcal{C}}$, if we sett $t_{\mathfrak{C}}(N)=\ln N-f_{\mathcal{C}}$ and denote by $p$ the projection $p(\hat{\alpha}, z)=\hat{\alpha}$, we have

$$
\hat{\mathscr{G}}^{n(N, \hat{\alpha})}(\hat{\alpha})=p \Phi_{t_{\mathcal{C}}(N)}(\hat{\alpha}, 0) .
$$

Let us denote by $A_{\Phi}=p^{-1} \hat{A}$, so that $\hat{\mathscr{G}}^{n(N, \hat{\alpha})}(\hat{\alpha}) \in \hat{A}$ iff $\Phi_{t_{\mathfrak{e}}(N)}(\hat{\alpha}, 0) \in A_{\Phi}$. For each $\varepsilon>0$, by absolute continuity of the integral, there exists $\delta^{\prime}>0$ and $\hat{A}=\hat{A}\left(\delta^{\prime}\right)$ so that $\mu_{3}\left(A_{\Phi}\right) \leq \varepsilon$. Hence, thickening $U_{\mathcal{e}}$ slightly, i. e. considering $U_{\mathcal{e}}^{\delta_{\mathfrak{e}}}=U_{\mathcal{e}} \times\left[0, \delta_{\mathcal{e}}\right)$ for some small $\delta_{\mathfrak{e}}$, as in the proof of [SU08, and exploiting mixing of the flow $\left\{\Phi_{t}\right\}_{t \in \mathbb{R}}$, there exists $N_{\mathcal{C}}$ so that for each $N \geq N_{\mathcal{C}}$

$$
\begin{aligned}
\mu_{2}\left\{\hat{\alpha} \in \mathcal{C}: \hat{\mathscr{G}}^{n(N, \hat{\alpha})}(\hat{\alpha}) \notin \hat{A}\right\} & \geq \frac{\mu_{3}\left(U_{\mathcal{C}}^{\delta_{\mathcal{e}}} \cap \Phi_{-t_{\mathcal{C}}(N)}\left(A_{\Phi}^{C}\right)\right)}{\delta_{\mathcal{C}}} \\
& \geq(1-\varepsilon)\left(1-\mu_{3}\left(A_{\Phi}\right)\right) \mu_{2}\left(U_{\mathcal{C}}\right) .
\end{aligned}
$$

\footnotetext{
${ }^{4}$ We recall that $f_{\mathcal{C}}=\sup _{\hat{\alpha} \in \mathcal{C}} f(\hat{\alpha})$ where $f=\lim _{n} f_{n}$ and $f_{n}(\hat{\alpha})=\ln q_{2 n}(\hat{\alpha})-S_{\hat{\alpha}}(\varphi, n)$ and we refer to SU08 for more comments of $f$ and the proof of the existence of the limit $f$.
} 
Summing over the finitely many $\mathcal{C}$ involved and choosing $\varepsilon$ so that $(1-\varepsilon)^{3} \geq(1-\delta)$ and $N_{0}=\max _{\mathcal{C}} N_{\mathcal{e}}$, this concludes the proof.

A.2. On the proof of Theorem 3, Let us sketch briefly how to obtain Theorem 3 from Theorem 1, SU08. The only differences between the two theorems are the following. First the measure considered is the 2-dimensional Lebesgue measure Leb, while Theorem 1 in SU08 is stated for the product $\mathscr{G} \times \lambda$ of the Gauss measure and $\lambda$, Lebesgue measure. Then the entries considered are $a_{n(N)+k}$ for $|k| \leq M$ instead than only $a_{n(N)+k}$ for $0<k \leq M$. Furthermore, $n$ is required to be even.

The first two differences require easy modifications. It was already remarked in SU08 that Theorem 1, SU08 holds for any absolutely continuous measure. To consider also $a_{n(N)+k}$ with $-M \leq k \leq 0$ it is enough to substitute the cylinder $C_{N}$ in (24), SU08 with $C_{M}:=\hat{\mathscr{G}}^{M-1} \hat{\mathcal{C}}\left(\left[c_{M} c_{M-1} \ldots, c_{0}, c_{-1}, \ldots, c_{-M}\right]\right)$ (we remark that here $M$ plays the role of $N$ in $[\mathbf{S U 0 8}$ ).

In order to have a limiting distributions when only even $n$ are considered (a choice which simplifies our analysis), it is necessary to use a slightly different special flow than the one in $\S 2$ of [SU08]. Instead than the base transformation $\hat{\mathscr{G}}$, consider the transformation $\hat{\mathscr{G}}^{2}$ and substitute the roof function $\varphi$ in (6) of [SU08] with

$$
\varphi(\hat{\alpha})=\ln \left(a_{1}+\frac{1}{a_{0}+\frac{1}{a_{-1}+\ldots}}\right)+\ln \left(a_{2}+\frac{1}{a_{1}+\frac{1}{a_{0}+\ldots}}\right) .
$$

In this way, Lemma 1 of [SU08] holds for $f_{n}(\hat{\alpha})=\ln q_{2 n}(\hat{\alpha})-S_{n}(\varphi, \hat{\alpha})$. The proof that the suspension flow under this new $\varphi$ is mixing proceeds as in $\S 4$ in [SU08: one can explicitly write the equations of local stable and unstable manifolds and check that they are non-integrable.

\section{References}

[HL30] G. H. Hardy and J. E. Littlewood, Some problems of diophantine approximation: a series of cosecants, Bulletin of the Calcutta Mathematica Society 20 (1930), no. 3, 251-266.

[Khi35] A. Ya. Khinchin, Continued fractions, The University of Chicago Press, 1935.

[Shi96] A. N. Shiryaev, Probability, Graduate Texts in Mathematics 95, Springer-Verlag, 1996, Second Edition.

[Sin94] Ya. G. Sinai, Topics in ergodic theory, Princeton University Press, 1994.

[SU08] Yakov G. Sinai and Corinna Ulcigrai, A renewal type limit theorem for the gauss map and continued fractions, Ergodic Theory and Dynamical Systems 28 (2008), no. 2, 643-655.

[Ulc07] Corinna Ulcigrai, Mixing for suspension flows over interval exchange tranformations, Ergodic Theory and Dynamical Systems 27 (2007), no. 3, 991-1035.

Mathematics Department, Princeton University, Princeton, New Jersey 08544, USA Landau Institute for Theoretical Physics, Moscow, Russia

E-mail address: sinai@math.princeton.edu

School of Mathematics, University of Bristol, Bristol BS8 1TW, United Kingdom

E-mail address: corinna.ulcigrai@bristol.ac.uk 